\title{
High-sensitivity maps of molecular ions in L1544
}

\section{Deuteration of $\mathrm{N}_{2} \mathrm{H}^{+}$and $\mathrm{HCO}^{+}$and primary evidence of $\mathrm{N}_{2} \mathrm{D}^{+}$depletion $\star$}

\author{
E. Redaelli, L. Bizzocchi, P. Caselli, O. Sipilä, V. Lattanzi, B. M. Giuliano, and S. Spezzano
}

\begin{abstract}
Centre for Astrochemical Studies, Max-Planck-Institut für extraterrestrische Physik, Gießenbachstraße 1, 85749 Garching bei München, Germany

e-mail: eredaelli@mpe.mpg.de
\end{abstract}

Received 20 February 2019 / Accepted 18 July 2019

\begin{abstract}
Context. The deuterium fraction in low-mass prestellar cores is a good diagnostic indicator of the initial phases of star formation, and is also a fundamental quantity to infer the ionisation degree in these objects.

Aims. With the analysis of multiple transitions of $\mathrm{N}_{2} \mathrm{H}^{+}, \mathrm{N}_{2} \mathrm{D}^{+}, \mathrm{HC}^{18} \mathrm{O}^{+}$, and $\mathrm{DCO}^{+}$we are able to determine the molecular column density maps and the deuterium fraction in $\mathrm{N}_{2} \mathrm{H}^{+}$and $\mathrm{HCO}^{+}$toward the prototypical prestellar core $\mathrm{L} 1544$. This is the preliminary step to derive the ionisation degree in the source.

Methods. We used a non-local thermodynamic equilibrium (non-LTE) radiative transfer code combined with the molecular abundances derived from a chemical model to infer the excitation conditions of all the observed transitions. This allowed us to derive reliable maps of the column density of each molecule. The ratio between the column density of a deuterated species and its nondeuterated counterpart gives the sought-after deuteration level.

Results. The non-LTE analysis confirms that, for the molecules analysed, higher-J transitions are characterised by excitation temperatures that are $\approx 1-2 \mathrm{~K}$ lower than those of the lower-J transitions. The chemical model that provides the best fit to the observational data predicts the depletion of $\mathrm{N}_{2} \mathrm{H}^{+}$and to a lesser extent of $\mathrm{N}_{2} \mathrm{D}^{+}$in the innermost region. The peak values for the deuterium fraction that we find are $\mathrm{D} / \mathrm{H}_{\mathrm{N}_{2} \mathrm{H}^{+}}=0.26_{-0.14}^{+0.15}$ and $\mathrm{D} / \mathrm{H}_{\mathrm{HCO}^{+}}=0.035_{-0.012}^{+0.015}$, in good agreement with previous estimates in the source.
\end{abstract}

Key words. ISM: clouds - ISM: molecules - ISM: abundances - radio lines: ISM - stars: formation

\section{Introduction}

Deuterium was formed during the primordial nucleosynthesis in the first minutes after the Big Bang with a fractional abundance of $\approx 1.5 \times 10^{-5}$ (Linsky 2003). However, enhancements of this value of several orders of magnitude have been found both in different environments of the interstellar medium (ISM) and in several components of the solar system (Ceccarelli et al. 2014, and references therein). This has led to the idea of using the deuteration ratio $\mathrm{D} / \mathrm{H}$ (the ratio between the abundance of a D-bearing molecule and its hydrogenated isotopologue) as a diagnostic tool to investigate the star formation process, and ultimately to understand how our own solar system was formed (Ceccarelli et al. 2014).

Prestellar cores represent the early phases of low-mass star formation (André et al. 2014). These are dense (central volume densities $\left.n \gtrsim 10^{5} \mathrm{~cm}^{-3}\right)$ and cold $(T \lesssim 10 \mathrm{~K})$ fragments of molecular clouds that are on the verge of gravitational collapse but still have not formed any central object (Keto \& Caselli 2008). Prestellar cores are known to exhibit some of the highest levels of deuteration $(\mathrm{D} / \mathrm{H}>0.1$, Crapsi et al. 2005; Pagani et al. 2007), because their physical conditions greatly favour the deuteration processes. D-fractionation (i.e. the inclusion of a D-atom in hydrogenated species) is indeed driven by

\footnotetext{
* This work is based on observations carried out with the IRAM $30 \mathrm{~m}$ Telescope. IRAM is supported by INSU/CNRS (France), MPG (Germany) and IGN (Spain).
}

the reaction:

$\mathrm{H}_{3}^{+}+\mathrm{HD} \leftrightharpoons \mathrm{H}_{2} \mathrm{D}^{+}+\mathrm{H}_{2}$,

which proceeds more efficiently from left to right as the temperature decreases (assuming a low ortho-to-para $\mathrm{H}_{2}$ ratio; e.g. Pagani et al. 1992) due to the lower zero-point energy of $\mathrm{H}_{2} \mathrm{D}^{+}$, the progenitor of all the deuterated species in regions where the temperature is $\$ 30 \mathrm{~K}$ (Dalgarno \& Lepp 1984). Thus, in cold environments, the increased abundance of $\mathrm{H}_{2} \mathrm{D}^{+}$and of the doubly and triply deuterated forms of $\mathrm{H}_{3}^{+}$rapidly leads to higher $\mathrm{D} / \mathrm{H}$ ratios in all molecules produced by the reactions with the various $\mathrm{H}_{3}^{+}$isotopologues. This process is also favoured by another mechanism. The $\mathrm{H}_{3}^{+}$main destruction route is its reaction with CO. Above $n \approx$ a few $10^{4} \mathrm{~cm}^{-3}$, CO and other C- and O-bearing species catastrophically freeze onto the dust grain surfaces, leading to their depletion from the gas phase; in regions where the abundance of neutral species reacting with $\mathrm{H}_{3}^{+}$is reduced, D-fractionation is enhanced (Dalgarno \& Lepp 1984). In prestellar cores, where the depletion fraction of $\mathrm{CO}$ is $>90 \%$ in the central 6000-8000 AU (Caselli et al. 1999; Bacmann et al. 2002), the D-fraction in $\mathrm{NH}_{3}$ reaches levels as high as $40 \%$ (Crapsi et al. 2005).

A quantity that plays a crucial role in driving the timescales of star formation is the ionisation degree, that is the ratio between the free electron density $n(e)$ and the gas density $n\left(\mathrm{H}_{2}\right)[x(e)=$ $\left.n(e) / n\left(\mathrm{H}_{2}\right)\right]$ (McKee 1989). This factor determines the timescale for the ambipolar diffusion of neutrals across the magnetic field 
Table 1. Main properties of the lines observed at the IRAM $30 \mathrm{~m}$ telescope.

\begin{tabular}{ccccccc}
\hline \hline Line & $v(\mathrm{GHz})^{(a)}$ & $\Delta V_{\text {ch }}\left(\mathrm{km} \mathrm{s}^{-1}\right)$ & $\theta_{\text {beam }}\left(^{\prime \prime}\right)$ & $F_{\text {eff }} / B_{\text {eff }}$ & Int. time $(\mathrm{h}){ }^{(b)}$ & Ref. \\
\hline $\mathrm{N}_{2} \mathrm{H}^{+}(1-0)$ & 93.173 & 0.06 & 27 & $0.95 / 0.81$ & 2.8 & 1 \\
$\mathrm{~N}_{2} \mathrm{H}^{+}(3-2)$ & 279.512 & 0.02 & 11 & $0.87 / 0.49$ & 11.3 & 1 \\
$\mathrm{~N}_{2} \mathrm{D}^{+}(1-0)$ & 77.109 & 0.08 & 34 & $0.95 / 0.82$ & 7.5 & 2 \\
$\mathrm{~N}_{2} \mathrm{D}^{+}(2-1)$ & 154.217 & 0.04 & 16 & $0.93 / 0.72$ & 6.7 & 2 \\
$\mathrm{~N}_{2} \mathrm{D}^{+}(3-2)$ & 231.322 & 0.03 & 12 & $0.92 / 0.59$ & 9.4 & 2 \\
$\mathrm{DCO}^{+}(1-0)$ & 72.039 & 0.20 & 36 & $0.95 / 0.82$ & 7.1 & 3 \\
$\mathrm{DCO}^{+}(2-1)$ & 144.077 & 0.04 & 17 & $0.93 / 0.73$ & 6.6 & 3 \\
$\mathrm{DCO}^{+}(3-2)$ & 216.113 & 0.03 & 12 & $0.94 / 0.63$ & 2.8 & 3 \\
$\mathrm{HC}^{18} \mathrm{O}^{+}(1-0)$ & 85.162 & 0.07 & 31 & $0.95 / 0.81$ & 10.2 & 4 \\
\hline
\end{tabular}

Notes. ${ }^{(a)}$ The references for the rest frequencies are found in Table A.1. ${ }^{(b)}$ Total observing time.

References. References used for the collisional coefficients (see Sect. 3.2): (1) Lique et al. (2015); (2) scaled from Lique et al. (2015); (3) Pagani et al. (2012); (4) scaled from Flower (1999).

lines, which is the main process that allows the gravitational collapse of magnetised cores (Mouschovias \& Spitzer 1976). In molecular clouds, the deuteration fraction is in general considered a good indicator of the ionisation degree (Guelin et al. 1977; Caselli et al. 1998, 2002a; Dalgarno 2006), as the D-fraction in species such as $\mathrm{HCO}^{+}$is a function of $x(e)$. As a consequence, accurate measurements of the $\mathrm{D} / \mathrm{H}$ ratio allow the ionisation degree in prestellar cores to be determined. In particular, $\mathrm{N}_{2} \mathrm{H}^{+}$ and $\mathrm{HCO}^{+}$represent ideal tracers (Caselli et al. 1998; Caselli 2002); these molecules - and their deuterated counterparts - are abundant in low-mass cores. It has been shown that $\mathrm{N}_{2} \mathrm{H}^{+}$is usually highly concentrated in the central parts, whereas $\mathrm{HCO}^{+}$ traces the outer layers (e.g. Punanova et al. 2018). Combined, they can provide information on the ionisation degree in the whole source.

L1544 is one of the most studied low-mass prestellar cores. Its vicinity ( $d \approx 135 \mathrm{pc}$, Schlafly et al. 2014) and bright continuum emission at mm and sub-mm wavelengths (Ward-Thompson et al. 1999; Doty et al. 2004; Spezzano et al. 2016) make it the ideal environment to study the early stages of low-mass star formation. The central part of the core is cold $(T \approx 6 \mathrm{~K}$, Crapsi et al. 2007) and dense $\left(n>10^{6} \mathrm{~cm}^{-3}\right.$, Tafalla et al. 2002; Keto \& Caselli 2010). It is centrally peaked and shows signatures of contraction (Caselli et al. 2002b, 2012; Lee et al. 2001), suggesting that it is on the verge of gravitational collapse. In the last $20 \mathrm{yr}$, a vast observational campaign combined with theoretical efforts has led to a deep knowledge of its physical and chemical structure (see e.g. Crapsi et al. 2007; Caselli et al. 2012; Keto et al. 2015; Spezzano et al. 2017). Previous works (Caselli et al. 1998, 2002a) have already investigated the deuteration level and ionisation fraction in this source, using the telescope capabilities available at that time. We now have access to new high-sensitivity maps of $\mathrm{N}_{2} \mathrm{H}^{+}, \mathrm{N}_{2} \mathrm{D}^{+}, \mathrm{HC}^{18} \mathrm{O}^{+}$, and $\mathrm{DCO}^{+}$across the whole core performed with the on-the-fly method. For the first time, we can analyse the first three rotational transitions of $\mathrm{N}_{2} \mathrm{D}^{+}$and $\mathrm{DCO}^{+}$and the $\mathrm{N}_{2} \mathrm{H}^{+}(1-0)$ and (3-2) lines ${ }^{1}$ simultaneously. In this paper we report the deuteration fraction maps of $\mathrm{N}_{2} \mathrm{H}^{+}$and $\mathrm{HCO}^{+}$in $\mathrm{L}_{1544}$, obtained with a detailed non-LTE modelling of the molecular spectra at the centre of the core. In a following work (Paper II) we will use the results presented here to accurately derive the ionisation fraction map of L1544.

1 The $\mathrm{N}_{2} \mathrm{H}^{+}$(2-1) transition is difficult to observe (although not impossible, Daniel et al. 2007), because it falls in the vicinity of a strong atmospheric line.
In Sect. 2 we present the observations. The analysis is described in Sect. 3, divided into chemical modelling (Sect. 3.1), non-LTE modelling (Sect. 3.2), and column density derivation (Sect. 3.3). We discuss the results in Sect. 4, and draw a summary of the work in Sect. 5.

\section{Observations}

All the data presented in this paper were collected with the IRAM $30 \mathrm{~m}$ single dish telescope. We used the four receivers of the frontend EMIR (E0, E1, E2 and E3), which allow us to cover the required frequency range. The $\mathrm{N}_{2} \mathrm{H}^{+}(1-0)$ data were acquired in summer 2015. Observations of $\mathrm{N}_{2} \mathrm{H}^{+}(3-2), \mathrm{N}_{2} \mathrm{D}^{+}$ $(1-0),(2-1)$, and $(3-2), \mathrm{HC}^{18} \mathrm{O}^{+}(1-0)$, and $\mathrm{DCO}^{+}(1-0),(2-1)$, and (3-2) were performed during winter 2015/2016 using four different spectral setups. The VESPA backend, with a spectral resolution of $20 \mathrm{kHz}$, was used for all data except for the $\mathrm{DCO}^{+}$ (1-0) transition, which was acquired with the FTS backend (resolution: $50 \mathrm{kHz}$ ). In all the sessions, the pointing was frequently checked on nearby planets or bright quasars, and found to be accurate within $2^{\prime \prime}$ and $5^{\prime \prime}$ (at the high and low frequencies, respectively). In order to encompass the whole core, all the transitions were acquired in the on-the-fly (OTF) map mode with a $2^{\prime} \times 2^{\prime}\left(\approx 0.08 \times 0.08 \mathrm{pc}^{2}\right)$ footprint centred at the L1544 dust peak $\left(\right.$ RA $(J 2000)=05^{\mathrm{h}} 04^{\prime} 17^{\prime \prime} .21$, Dec $(J 2000)=25^{\circ} 10^{\prime} 42^{\prime \prime} .8$, Ward-Thompson et al. 1999). The $\mathrm{N}_{2} \mathrm{H}^{+}(1-0)$ data, observed in a different session, consist of a $4^{\prime} \times 4^{\prime}\left(\approx 0.16 \times 0.16 \mathrm{pc}^{2}\right)$ map across the dust peak.

The spectra, originally calibrated to antenna temperature $\left(T_{\mathrm{A}}^{*}\right)$, were converted to main beam temperature $\left(T_{\mathrm{MB}}\right)$ using the tabulated values for the $30 \mathrm{~m}$ forward efficiency $\left(F_{\text {eff }}\right)$ and main beam efficiency $\left(B_{\text {eff }}\right)$. The main features of the observed lines, such as their frequency, angular resolution $\left(\theta_{\text {beam }}\right)$, and spectral resolution $\left(\Delta V_{\mathrm{ch}}\right)$, are summarised in Table 1 .

In addition to these data, we also make use of a single pointing observation of the $\mathrm{HCO}^{+}(1-0)$ line performed with the FCRAO $14 \mathrm{~m}$ telescope towards the dust peak of L1544. These data have a spectral resolution of $0.07 \mathrm{~km} \mathrm{~s}^{-1}$ and an angular resolution of 55", and were previously published in Tafalla et al. (1998). We refer to their paper for further details.

Results. Figures 1 and 2 show the observed spectra at the millimetre dust peak for each transition (in black). Considering only the emission-free channels, we computed the mean rms per channel for each transition, obtaining: $0.05 \mathrm{~K}$ for $\mathrm{N}_{2} \mathrm{D}^{+}(1-0)$, $0.18 \mathrm{~K}$ for $\mathrm{N}_{2} \mathrm{D}^{+}(2-1), 0.26 \mathrm{~K}$ for $\mathrm{N}_{2} \mathrm{D}^{+}(3-2), 0.14 \mathrm{~K}$ for 

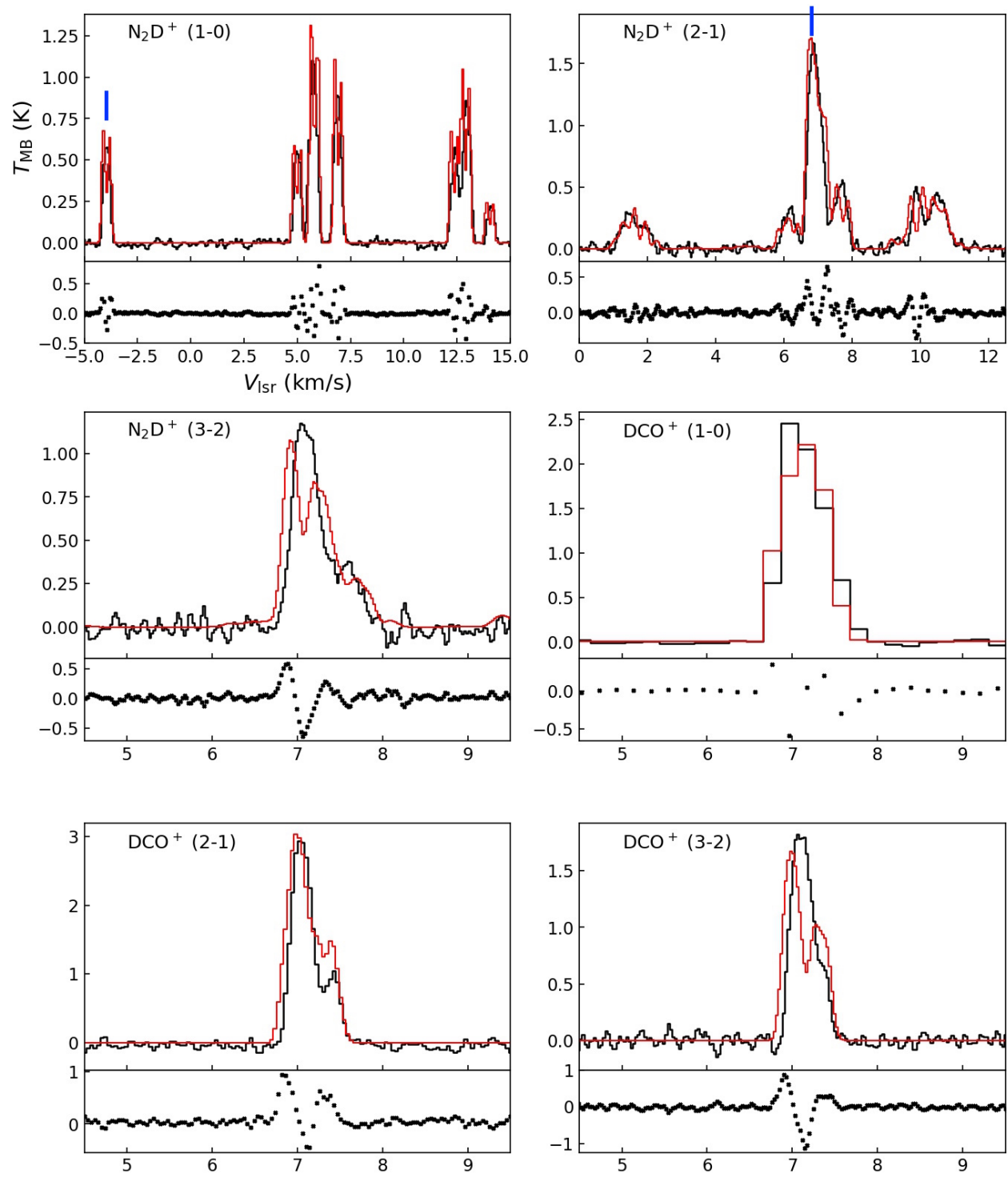

Fig. 1. Observed spectra at the dust peak of $\mathrm{N}_{2} \mathrm{D}^{+}$and $\mathrm{DCO}^{+}$, with the original angular resolution (in black histograms). The transition is labelled in the upper-left corner of each panel. In red, the best-fit solution found with MOLLIE is shown overlaying the observations (see Sect. 3.2). Underneath each line we present the residuals of the fit (difference between the model and the observation). The vertical blue bars show which hyperfine component has been used to derive the molecular column density, when the hyperfine structure has not been neglected.

$\mathrm{N}_{2} \mathrm{H}^{+}(1-0), 0.32 \mathrm{~K}$ for $\mathrm{N}_{2} \mathrm{H}^{+}(3-2), 0.04 \mathrm{~K}$ for $\mathrm{HC}^{18} \mathrm{O}^{+}(1-$ $0), 0.08 \mathrm{~K}_{\text {for }} \mathrm{DCO}^{+}(1-0), 0.07 \mathrm{~K}$ for $\mathrm{DCO}^{+}(2-1)$, and $0.23 \mathrm{~K}$ for $\mathrm{DCO}^{+}(3-2)$. Thus, at the dust peak the observed spectra have a signal-to-noise ratio $(\mathrm{S} / \mathrm{N})$ ranging from $\approx 4.0$ to $\approx 40.0$. Figure 3 shows the integrated intensity maps for each transition, computed integrating over all the hyperfine components when present.

\section{Analysis}

Our main goal is to derive the deuterium fraction across the source, and for this aim we need reliable measurements of the column density for each isotopologue. In principle, the knowledge of multiple transitions of a species can allow both its column density and excitation condition (i.e. the line excitation temperature, $T_{\mathrm{ex}}$ ) to be determined. This approach, implemented in the population diagram method (Goldsmith \& Langer 1999), has been extensively used in the literature, but it is valid only in the assumption that all the lines share the same $T_{\mathrm{ex}}$. This assumption extends to all the hyperfine components, when present. However, we know from previous studies (Daniel et al. 2006, 2013) that this is often not the case for the molecules considered here. There are multiple possible reasons for this, such as selective photon trapping in very crowded hyperfine structure, or a significant difference in critical density for the different rotational transitions of the same molecule (the Einstein coefficient $A$ scales with the third power of the frequency). In this case, the column density and the excitation temperatures of the different transitions are degenerate parameters, and cannot be constrained simultaneously. Therefore, an approach that does not assume LTE is needed to determine the $T_{\mathrm{ex}}$ of each transition and thus the molecular column density.

A full non-LTE analysis is on the other hand not feasible on maps, because it requires the knowledge of the full threedimensional temperature and density structure of the core. For L1544, however, Keto et al. (2015) developed a one-dimensional physical model based on a collapsing Bonnor-Ebert sphere that provides a good fit to several molecules at the dust peak (Caselli et al. 2012, 2017; Bizzocchi et al. 2013; Redaelli et al. 2018). We thus decided to model the spectra at the dust peak of the core with a full non-LTE analysis. In this way, we are able to constrain the column density of each molecule at the dust peak. At the same time, we can use the equations of radiative transfer to derive $N_{\text {col }}$ in the whole map. Since these two approaches must return the same result at the centre of the core, the $T_{\mathrm{ex}}$ (which is involved in the radiative transfer equations) is treated as a free 

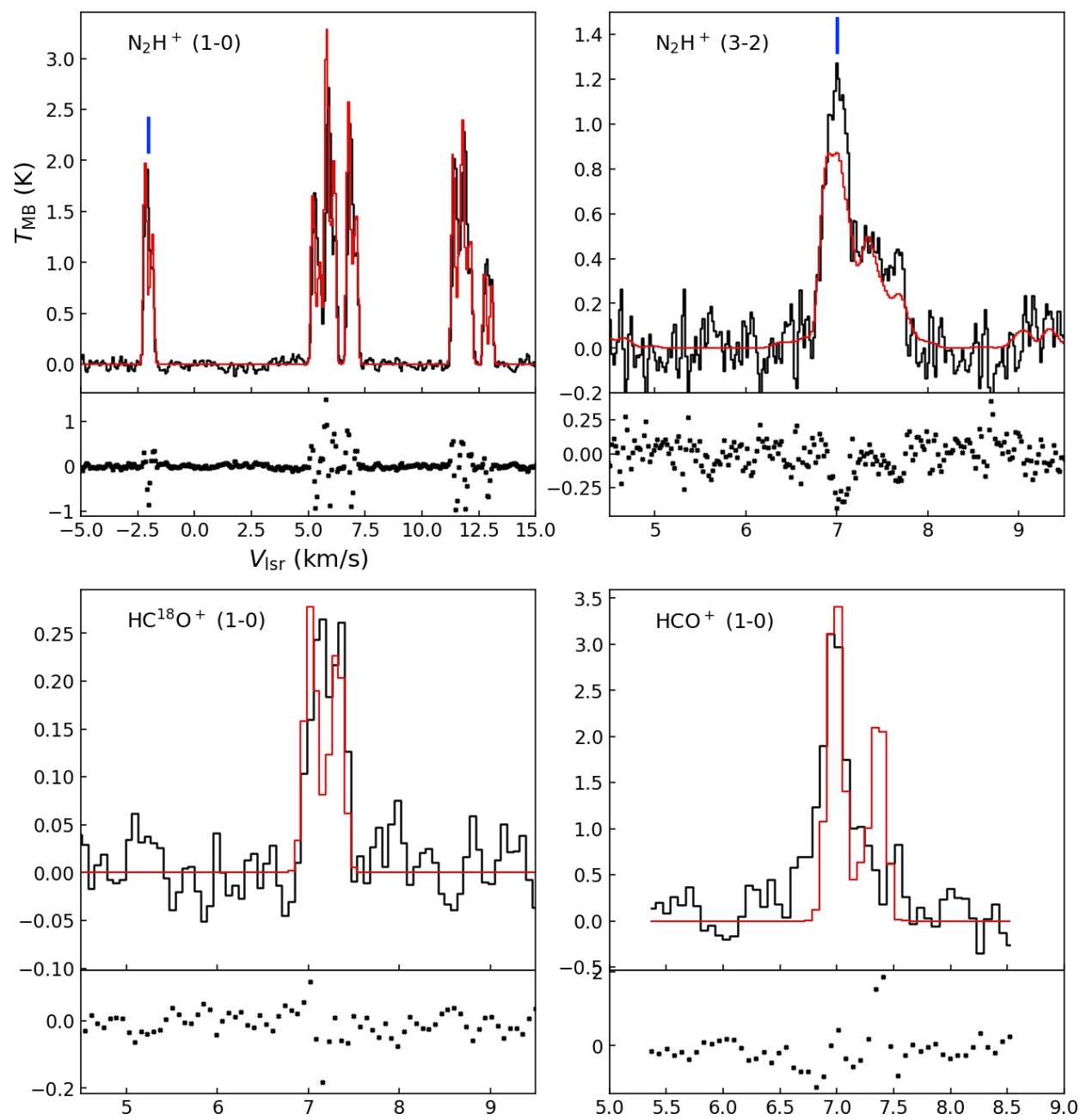

Fig. 2. As in Fig. 1, but for $\mathrm{N}_{2} \mathrm{H}^{+}, \mathrm{HC}^{18} \mathrm{O}^{+}$ and $\mathrm{HCO}^{+}$transitions.

parameter to be tuned in order to obtain the expected agreement of the two methods. The non-LTE modelling allows us to break the degeneracy between $N_{\text {col }}$ and $T_{\text {ex }}$ mentioned in the previous paragraph. In the following sections we provide a detailed description of the required steps.

\subsection{Chemical models}

The non-LTE modelling of line emission requires the knowledge of molecular abundance $\left(X_{\mathrm{mol}}\right)$ profiles. To derive these, we used the gas-grain chemical model discussed in Sipilä et al. (2015a,b), which has recently received an extensive update (Sipilä et al. 2019). Briefly, we employ the static physical core model from Keto et al. (2015), divided into concentric shells, and solve the chemical evolution separately in each shell. We then combine the results at different time steps to produce radius-dependent abundance profiles for the molecules of interest. The adopted initial conditions are summarised in Table 2. More information on typical model parameters can be found for instance in Sipilä et al. (2015a).

In this work we considered five different time steps: $t=$ $10^{4} \mathrm{yr}, t=5 \times 10^{4} \mathrm{yr}, t=10^{5} \mathrm{yr}, t=5 \times 10^{5} \mathrm{yr}$, and $t=10^{6} \mathrm{yr}$. We tested the influence of external UV radiation by considering three different visual extinction values for the cloud embedding the core: $A_{\mathrm{V}}=1,2$, or 5 mag. This amounts to a total of 15 modelled abundance profiles per molecule. We show the complete set of profiles in Appendix B. Simulated lines were produced based on these profiles as described in the following section.
Table 2. Initial abundances (with respect to the total hydrogen number density $n_{\mathrm{H}}$ ) used in the chemical modelling.

\begin{tabular}{cc}
\hline \hline Species & Abundance \\
\hline $\mathrm{H}_{2}$ & $5.00 \times 10^{-1}$ \\
$\mathrm{He}$ & $9.00 \times 10^{-2}$ \\
$\mathrm{HD}$ & $1.60 \times 10^{-5}$ \\
$\mathrm{C}^{+}$ & $1.20 \times 10^{-4}$ \\
$\mathrm{~N}$ & $7.60 \times 10^{-5}$ \\
$\mathrm{O}$ & $2.56 \times 10^{-4}$ \\
$\mathrm{~S}^{+}$ & $8.00 \times 10^{-8}$ \\
$\mathrm{Si}^{+}$ & $8.00 \times 10^{-9}$ \\
$\mathrm{Na}^{+}$ & $2.00 \times 10^{-9}$ \\
$\mathrm{Mg}^{+}$ & $7.00 \times 10^{-9}$ \\
$\mathrm{Fe}^{+}$ & $3.00 \times 10^{-9}$ \\
$\mathrm{P}^{+}$ & $2.00 \times 10^{-10}$ \\
$\mathrm{Cl}^{+}$ & $1.00 \times 10^{-9}$ \\
\hline
\end{tabular}

Notes. The initial $\mathrm{H}_{2}$ ortho/para ratio is $1.0 \times 10^{-3}$.

\subsection{Non-LTE modelling at the dust peak}

The non-LTE approach was implemented using the radiative transfer code MOLLIE (Keto 1990; Keto et al. 2004), which allows us to derive synthetic spectra from a given physical model. MOLLIE is able to treat the line overlap that occurs in 

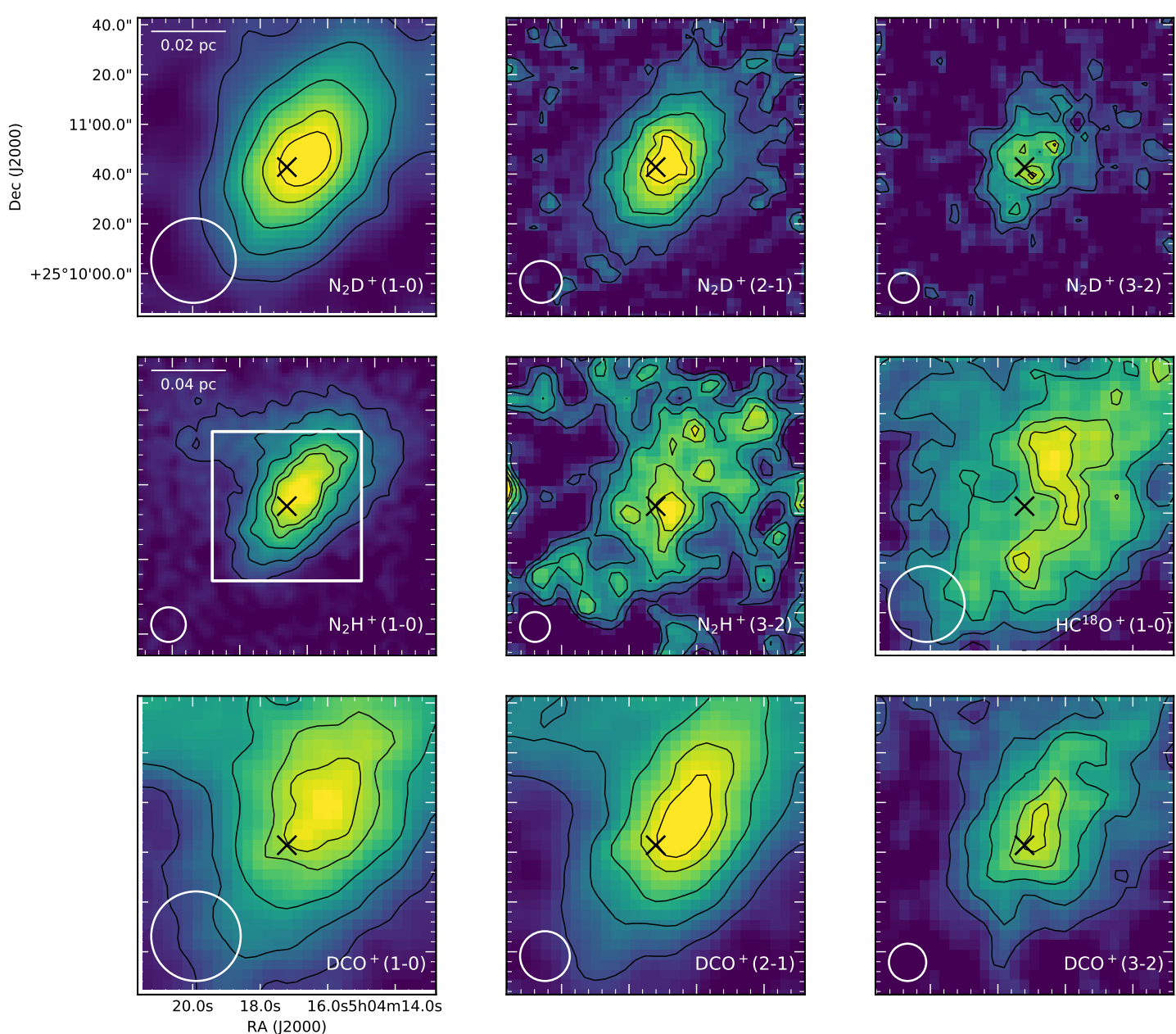

Fig. 3. Integrated intensity maps of all the observed transitions, which are labelled in the bottom-right corners. The contours show $20,40,60,80$, and $90 \%$ of the peak values, which are in order from top left to bottom right panels: $2.0,2.0,0.7,4.6,0.7,0.15,1.8,1.1,0.7 \mathrm{~K} \mathrm{~km} \mathrm{~s}^{-1}$. The black cross represents the dust peak position, and the white circle is the beam size. All the maps have the same size $\left(2^{\prime} \times 2^{\prime}\right)$, with the exception of $\mathrm{N}_{2} \mathrm{H}^{+}$ (1-0), which is $4^{\prime} \times 4^{\prime}$ : in this map the white rectangle shows the smaller coverage of the other transitions. Scalebars are shown in the top-left corners of the $\mathrm{N}_{2} \mathrm{D}^{+}(1-0)$ and $\mathrm{N}_{2} \mathrm{H}^{+}(1-0)$ maps.

a crowded hyperfine structure, such as those of $\mathrm{N}_{2} \mathrm{H}^{+}$and $\mathrm{N}_{2} \mathrm{D}^{+}$. As an output, it also provides the molecular column density, obtained by integrating the abundance profile multiplied by the gas density, eventually convolved to a desired beam size.

The physical model of the source, as already mentioned, is taken from Keto et al. (2015). It is an unstable quasi-equilibrium Bonnor-Ebert sphere, with a central density $n_{0} \approx 10^{7} \mathrm{~cm}^{-3}$ and a central temperature of 6.5 and $6.3 \mathrm{~K}$ for the gas and the dust, respectively. Figure 4 shows the profiles of the main quantities for this model. Recently, Chacon-Tanarro et al. (2019) proposed a new density and (dust) temperature model for L1544, analysing new millimetre continuum observations of L1544. However, the authors did not develop a new model for the velocity field, a crucial quantity to correctly reproduce molecular spectra. Therefore, we prefer to use the previous model, which also includes the velocity profile.

For the line radiative transfer, the collisional coefficients are of great importance, and we used the most recent ones. When they are available only for one isotopologue, we scaled them taking into account the different reduced mass of the collision system. The collisional coefficients for $\mathrm{N}_{2} \mathrm{H}^{+}$are taken from Lique et al. (2015), who computed them for the $\mathrm{N}_{2} \mathrm{H}^{+} / \mathrm{p}-\mathrm{H}_{2}$

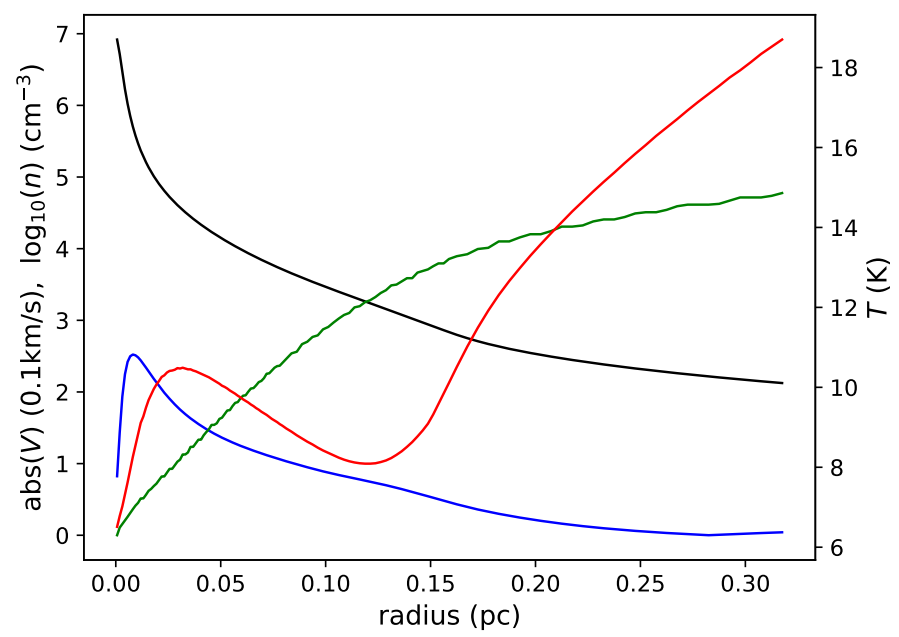

Fig. 4. Profiles of dust temperature (green), gas temperature (red), $\mathrm{H}_{2}$ volume density (black, in logarithmic scale), and velocity (blue, in units of $0.1 \mathrm{~km} \mathrm{~s}^{-1}$ ) for the L1544 model developed in Keto et al. (2015). The velocity in the model is negative (it represents contraction motions), but it is shown here as positive for improved readability. 
system. The rates for the $\mathrm{DCO}^{+} / \mathrm{p}-\mathrm{H}_{2}$ system were computed by Pagani et al. (2012), who take into account the hyperfine structure given by the deuterium nucleus. Finally, the collisions of $\mathrm{HCO}^{+}$with $\mathrm{p}-\mathrm{H}_{2}$ were published by Flower (1999), from which we scaled the coefficients for $\mathrm{HC}^{18} \mathrm{O}^{+}$. In all cases, the $\mathrm{o}-\mathrm{H}_{2}$ is neglected, which is a reasonable assumption given that the ortho-to-para ratio (OPR) in L1544 is found to be as low as OPR $=10^{-3}$ (Kong et al. 2015).

We tested all the 15 models described in Sect. 3.1. The abundance profiles produced by the chemical model often systematically overestimate or underestimate the observed fluxes, and need to be multiplied by a numerical factor to find a good agreement with the observations. However, MOLLIE requires too much computational time to allow a full sampling of the parameter space. For each molecule, we therefore proceeded as follows: first, we test all the original models, varying the evolutionary stage and the thickness of the surrounding cloud, and we select the one that best reproduces the trend of the different transitions (e.g. the ratio between the peak temperatures of the brightest component of each line). We then multiply the whole profile by a numerical factor, testing approximately five values until the observed fluxes are matched. This is considered the best fit to the observations. We want to highlight that our goal is to obtain a simultaneous fit for all molecules that is roughly consistent with the observations, and a full parameter-space search for the best possible fit within the model uncertainties is beyond the scope of the present paper. In the following text, we focus on the abundance profiles that provide the best-fit solution for each molecule. As an example, we present in Sect. 3.2.2 the resulting spectra from several of the 15 abundance profiles for $\mathrm{N}_{2} \mathrm{D}^{+}$.

\subsubsection{Best-fit solution for the observed molecules at the dust peak}

The best-fit solutions for each molecule are shown in red in Figs. 1 and 2 overlaying the observations. We also include here the analysis of a single-pointing observation of $\mathrm{HCO}^{+}(1-0)$ performed at the dust peak (see Sect. 2 for details). Despite the fact that a map for this transition is not available, it is important to check the behaviour of the chemical model for $\mathrm{HCO}^{+}$, the main isotopologue of its family. The observed spectrum of $\mathrm{HCO}^{+}$, together with the best fit from MOLLIE, is shown in the bottom-right panel of Fig. 2.

$\mathrm{N}_{2} \mathrm{H}^{+}$. The chemical network systematically underestimates the emission of $\mathrm{N}_{2} \mathrm{H}^{+}$, for any combination of chemical time and external visual extinction. We find that in general the spectra are best reproduced at late evolutionary times $\left(10^{6} \mathrm{yr}\right)$, when the molecules at the centre of the core start to be affected by depletion. This allows the observed ratio between the (1-0) and the (3-2) line to be reproduced. Our simulations show that varying the thickness of the external layer does not have a significant impact on the synthetic spectra due to the high critical density of $\mathrm{N}_{2} \mathrm{H}^{+}$whose emission arises only from the central regions ( $n_{\text {crit }} \gtrsim 10^{5} \mathrm{~cm}^{-3}$; see Shirley 2015). Furthermore, $\mathrm{N}_{2}$ is a late-type molecule that can take about ten times longer than CO to form (e.g. Hily-Blant et al. 2010). Therefore, the outer less dense envelope may not be rich enough in $\mathrm{N}_{2}$ for $\mathrm{N}_{2} \mathrm{H}^{+}$to form efficiently. The abundance profile for $t=10^{6} \mathrm{yr}$ and $A_{\mathrm{V}}=1 \mathrm{had}$ to be multiplied by 2.7 to match the observed fluxes.

$\mathrm{N}_{2} D^{+}$. Similar considerations to the ones made for the main isotopologue hold also for $\mathrm{N}_{2} \mathrm{D}^{+}$. The best fit is found for $t=10^{6} \mathrm{yr}$ and an external visual extinction of $A_{\mathrm{V}}=1 \mathrm{mag}$, even though this last parameter does not significantly affect the simulated spectra (see Sect. 3.2.2 for more details). The models again tend to underestimate the observed fluxes, and we had to increase the abundance profile by a factor of 3.0 to obtain a good agreement.

$\mathrm{HCO}^{+}$. In general, the original abundance profiles coming from the chemical network result in a better agreement with the observations of the $\mathrm{HCO}^{+}$isotopologues with respect to the $\mathrm{N}_{2} \mathrm{H}^{+}$ones. The best agreement for the main isotopologue is found for the model with $t=10^{6} \mathrm{yr}$ and an external visual extinction of $A_{\mathrm{V}}=5 \mathrm{mag}$, multiplied by a factor 0.5 . This model presents a high molecular abundance in the external layers of the cores $\left(X_{\text {mol }} \gtrsim 10^{-7}\right)$, which is important to correctly reproduce the strong blue asymmetry of the (1-0) line due to self-absorption (Tafalla et al. 1998).

$\mathrm{HC}^{18} \mathrm{O}^{+}$. In the chemical model, the abundance profile for the $\mathrm{HC}^{18} \mathrm{O}^{+}$is derived from that of $\mathrm{HCO}^{+}$using the scaling factor valid for the local ISM ${ }^{16} \mathrm{O} /{ }^{18} \mathrm{O}=557$ (Wilson 1999). This choice however leads to an underestimation of the observed flux. The best fit is found with the model at $t=10^{6} \mathrm{yr}$ and $A_{\mathrm{V}}=1 \mathrm{mag}$, multiplied by 6.0 . This late evolutionary stage is important to be able to reproduce the double-peak feature seen in the (1-0) spectrum, but we had to decrease the external visual extinction with respect to the main isotopologue. If the abundance of $\mathrm{HC}^{18} \mathrm{O}^{+}$is too high in the external layers of the core, as in the models with $A_{\mathrm{V}}=5 \mathrm{mag}$, the resulting synthetic spectra present a blue asymmetry due to the self-absorption, which is not seen in the observations.

$\mathrm{DCO}^{+}$. The three transitions of $\mathrm{DCO}^{+}$are best reproduced with the model at $t=10^{6} \mathrm{yr}$ and $A_{\mathrm{V}}=1 \mathrm{mag}$. In this case, we decrease the original abundance profile by $50 \%$ to match the observed peak temperatures.

The abundance profiles that provide the best-fit solution for each species are shown in Fig. 5, after the multiplication for the corrective factors. The adopted models and scaling factors, together with the molecular column density computed by MOLLIE at the centre of the core $\left(N_{\text {col }}^{\text {MOLLIE }}\right)$, are summarised in Table 3. It is not surprising that the chemical model results do not provide a perfect match to the observations, and that the various lines cannot be fitted with a single model. There are many sources of uncertainty, such as the source model (density and gas/dust temperature structures), chemical modelling parameters, and rate coefficients. Our model does not consider the self-shielding of $\mathrm{CO}$, which would affect the $\mathrm{HCO}^{+}$abundance in the outer core, for example. A detailed parameter-space exploration of the abundance profiles is beyond the scope of the present paper.

\subsection{2. $\mathrm{N}_{2} \mathrm{D}^{+}$depletion}

In this section, we analyse in detail the case of $\mathrm{N}_{2} \mathrm{D}^{+}$, showing the non-LTE radiative transfer results for 6 out of the 15 abundance profiles that we tested at the dust peak. The abundance profiles are all multiplied by a factor of 3.0 to allow an easier comparison with the observations. The obtained spectra are shown in Fig. 6 overlaying the observations.

The first four rows from the top in Fig. 6 show increasing evolutionary times, keeping the external extinction fixed to $A_{\mathrm{V}}=$ $1 \mathrm{mag}$. The difference between the observations and the model can be quantified by comparing the relative ratio of the peak temperatures of the three lines. In the observations, this ratio is $T_{\text {peak }}(1-0): T_{\text {peak }}(2-1): T_{\text {peak }}(3-2)=1: 1.5: 1.1$. In the models with the earlier times $\left(t=5 \times 10^{4}\right.$ and $\left.t=10^{5} \mathrm{yr}\right)$, the obtained ratios are 1:3.3:2.5 and 1:2.6:1.9, respectively. This means that these abundance profiles overestimate the (2-1) and (3-2) with 

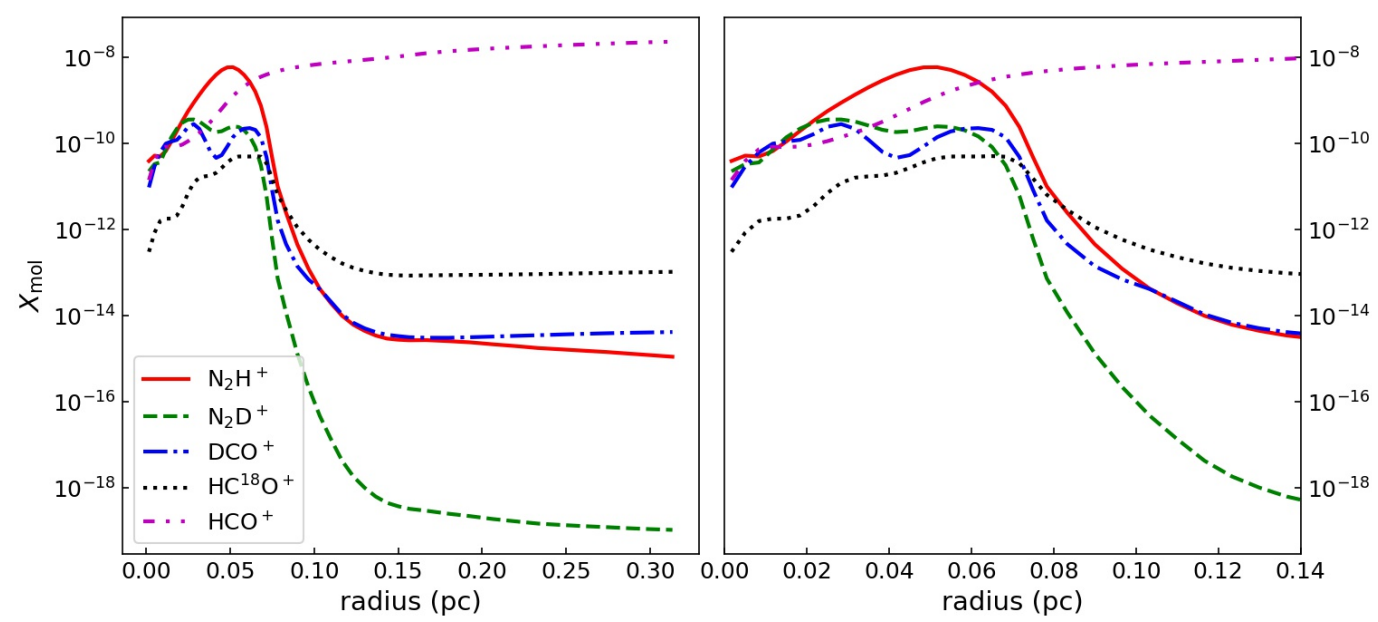

Fig. 5. Left panel: molecular abundances that provide the best fit to the spectra at the dust peak for the different species as a function of the core radius. Right panel: zoom-in of the molecular abundances in the inner $0.14 \mathrm{pc}$.

Table 3. Summary of the models that provide the best fit of each molecule, with the adopted scaling factor for the abundance profile.

\begin{tabular}{|c|c|c|c|c|c|}
\hline Molecule & $X_{\mathrm{col}}$ & $\begin{array}{c}\text { Scaling } \\
\text { factor }\end{array}$ & $\begin{array}{l}N_{\text {col }}^{\operatorname{MOLIE}}{ }^{(a)} \\
\left(10^{12} \mathrm{~cm}^{-2}\right)\end{array}$ & $\begin{array}{r}T_{\mathrm{ex}}^{(b)} \\
\text { line } \mathrm{e}^{(c)}\end{array}$ & $(\mathrm{K})$ \\
\hline $\mathrm{N}_{2} \mathrm{H}^{+}$ & $t=10^{6} \mathrm{yr}, A_{\mathrm{V}}=1$ & 2.7 & 17 & $\begin{array}{l}(1-0) \\
(3-2)\end{array}$ & $\begin{array}{l}5.6 \\
5.0\end{array}$ \\
\hline $\mathrm{N}_{2} \mathrm{D}^{+}$ & $t=10^{6} \mathrm{yr}, A_{\mathrm{V}}=1$ & 3.0 & 3.5 & $\begin{array}{l}(1-0) \\
(2-1) \\
(3-2)\end{array}$ & $\begin{array}{l}7.7 \\
5.0 \\
4.8\end{array}$ \\
\hline $\mathrm{HC}^{18} \mathrm{O}^{+}$ & $t=10^{6} \mathrm{yr}, A_{\mathrm{V}}=1$ & 6.0 & 0.18 & $(1-0)$ & 5.5 \\
\hline $\mathrm{DCO}^{+}$ & $t=10^{6} \mathrm{yr}, A_{\mathrm{V}}=1$ & 0.5 & 2.5 & $\begin{array}{l}(1-0) \\
(2-1) \\
(3-2)\end{array}$ & $\begin{array}{l}7.8 \\
5.8 \\
5.7\end{array}$ \\
\hline
\end{tabular}

Notes. ${ }^{(a)}$ Column density predicted by MOLLIE at the dust peak, computed integrating the molecular abundance, multiplied by the $\mathrm{H}_{2}$ volume density, along the line of sight and convolving to the beam size of the observation. ${ }^{(b)}$ Excitation temperature values for each line derived as described in the main text and used to derive the column density maps. ${ }^{(c)}$ The transitions are labelled with the rotational convention $\left(J-J^{\prime}\right)$, even when a single hyperfine component is used.

respect to the (1-0) line. In the later evolutionary stages $(t=$ $5 \times 10^{5}$ and $\left.t=10^{6} \mathrm{yr}\right)$, the ratios are $1: 1.2: 0.7$ and $1: 1.3: 0.8$, closer to the observed one. This trend can be understood by looking at the behaviour of the different abundance profiles in the inner $10000 \mathrm{AU}$, shown in Fig. 7. At earlier times, the $\mathrm{N}_{2} \mathrm{D}^{+}$ abundance peaks within the central 2000-3000 AU, where the molecular hydrogen density is $n\left(\mathrm{H}_{2}\right) \gtrsim 10^{5} \mathrm{~cm}^{-3}$. Since the critical densities of the (1-0), (2-1), and (3-2) transitions are $n_{\text {crit }}=$ $7.6 \times 10^{4}, 8.7 \times 10^{5}$, and $3.8 \times 10^{6} \mathrm{~cm}^{-3}$, respectively (Shirley $2015)$, this is also the region where the (2-1) and the (3-2) lines are emitting the most. At later evolutionary times, $\mathrm{N}_{2} \mathrm{D}^{+}$ is depleted and the abundance decreases by more than a factor of five for $r \lesssim 2000 \mathrm{AU}$. This is due to the fact that its precursor species, $\mathrm{N}_{2}$, starts to freeze-out onto dust grains, and as a consequence the formation rate of $\mathrm{N}_{2} \mathrm{D}^{+}$is reduced. The $\mathrm{N}_{2} \mathrm{D}^{+}$abundance peak moves outwards to $r \approx 5000 \mathrm{AU}$, where $n\left(\mathrm{H}_{2}\right) \approx 5 \times 10^{4} \mathrm{~cm}^{-3}$. Therefore, the (2-1) and (3-2) lines are less excited, while the (1-0) flux keeps rising. This is the first observational confirmation of $\mathrm{N}_{2} \mathrm{D}^{+}$partial depletion in cold and dense environments.

We now focus on the influence of the external embedding layer, keeping the evolutionary stage fixed $\left(t=10^{6} \mathrm{yr}\right)$ and varying the external visual extinction (see the lower three rows of Fig. 6). The synthetic spectra are not very sensitive to this change, both in the flux level and in the spectral profiles, especially concerning the (2-1) and (3-2) lines. This is again due to a combination of molecular abundance and excitation conditions. In fact, increasing $A_{\mathrm{V}}$ significantly changes the abundance only for $r \gtrsim 10000 \mathrm{AU}$, where the molecular hydrogen density is $n\left(\mathrm{H}_{2}\right) \lesssim 10^{4} \mathrm{~cm}^{-3}$. Therefore, the high-frequency transitions are not significantly affected by the change of $A_{\mathrm{V}}$.

\subsection{Column density and deuteration maps}

In the following, we describe how we derive the column density map of each species, knowing the $N_{\text {col }}$ value at the centre of the core from the non-LTE modelling, via the radiative transfer equations. The spectra of the analysed molecules greatly differ due to their opacity properties and hyperfine structure. For instance, we have optically thin transitions $\left(\mathrm{HC}^{18} \mathrm{O}^{+}(1-0)\right)$, or optically thick ones $\left(\mathrm{HCO}^{+}(1-0)\right)$. Some species present crowded hyperfine structures (such as $\mathrm{N}_{2} \mathrm{H}^{+}$and $\mathrm{N}_{2} \mathrm{D}^{+}$), while others show a much simpler profile (e.g. $\mathrm{DCO}^{+}$, where the hyperfine splitting due to the deuterium spin is not detectable at the 

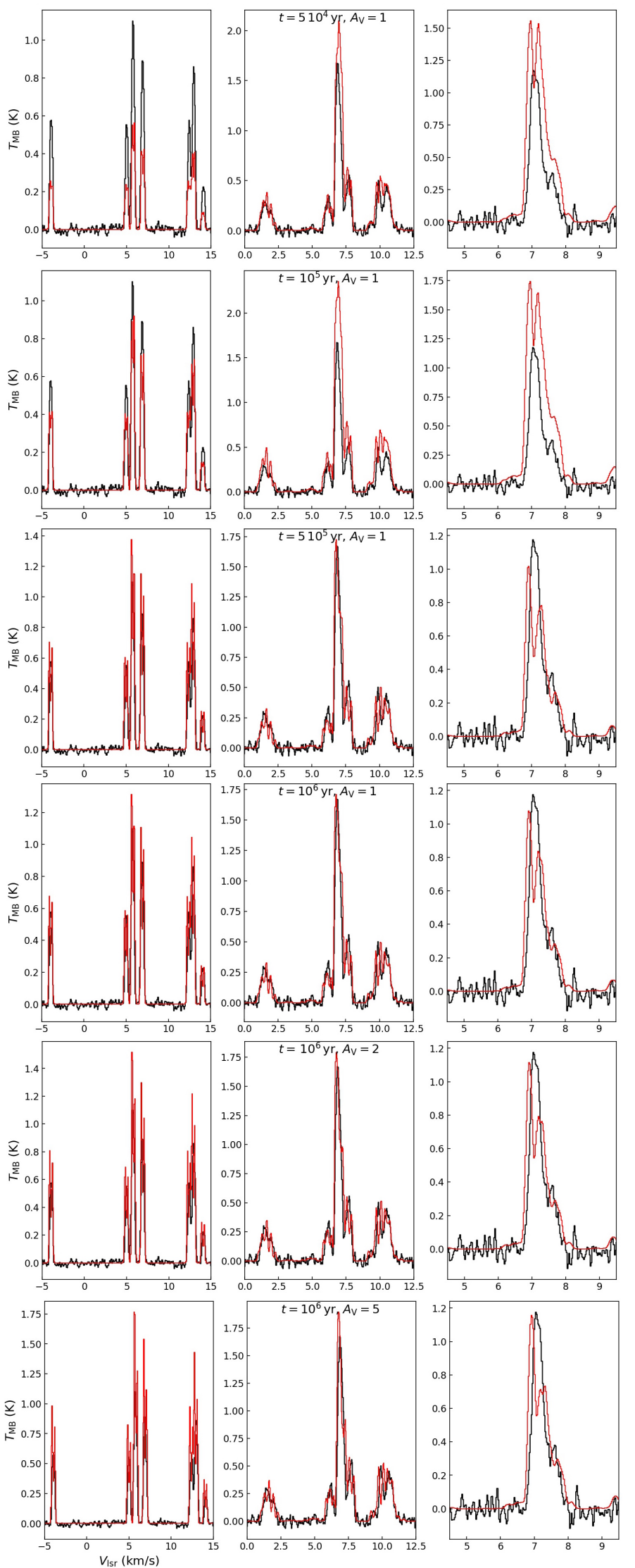

Fig. 6. Results of the line radiative transfer for six chemical models on the three transitions of $\mathrm{N}_{2} \mathrm{D}^{+}$, respectively (1-0), (2-1), and (3-2) from left to right in each panel. The synthetic spectra are shown with red histograms overlaying the observations (in black). Each model is labelled with its evolutionary stage and external visual extinction.

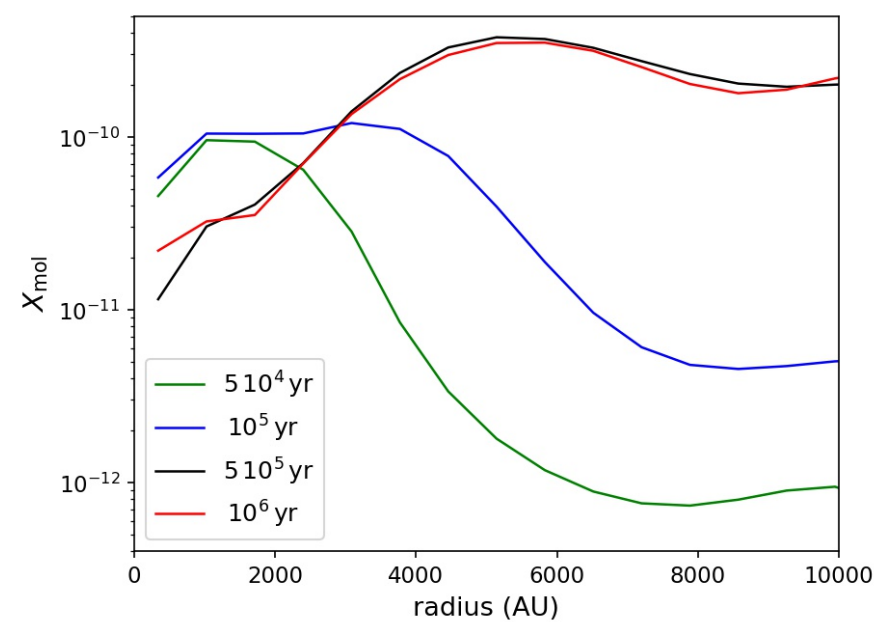

Fig. 7. Zoom-in of the central $10000 \mathrm{AU}$ of the $\mathrm{N}_{2} \mathrm{D}^{+}$abundances predicted by the chemical code for $A_{\mathrm{V}}=1 \mathrm{mag}$ at four evolutionary stages: $t=5 \times 10^{4} \mathrm{yr}$ (green), $t=10^{5} \mathrm{yr}$ (blue), $t=5 \times 10^{5} \mathrm{yr}$ (black), and $t=10^{6} \mathrm{yr}$ (red). The abundance profiles are multiplied by a factor of 3.0

given spectral resolution; Caselli \& Dore 2005). As a result, we tackle the problem of deriving the column density maps by developing a specific approach for each species individually. In all cases, we make use of the following well-known relations.

$T_{\mathrm{MB}}=\eta_{\mathrm{bf}}\left[J_{v}\left(T_{\mathrm{ex}}\right)-J_{v}\left(T_{\mathrm{bg}}\right)\right]\left(1-\mathrm{e}^{-\tau_{v}}\right)$,

and

$\tau_{v}=\sqrt{\frac{\ln 2}{16 \pi^{3}}} \frac{c^{3} A_{\mathrm{ul}} g_{\mathrm{u}}}{v^{3} Q\left(T_{\mathrm{ex}}\right) \Delta V} \mathrm{e}^{-\frac{E_{\mathrm{u}}}{k_{\mathrm{B}} T_{\mathrm{ex}}}}\left(\mathrm{e}^{\frac{h v}{k_{\mathrm{B}} T_{\mathrm{ex}}}}-1\right) N_{\text {col }}$.

Equation (2) describes the main beam temperature observed for a line with a given optical depth $\tau_{v}$ and a given excitation temperature $T_{\mathrm{ex}}$. The function $J_{v}$ is the equivalent Rayleigh-Jeans temperature, $T_{\mathrm{bg}}=2.73 \mathrm{~K}$ is the cosmic background temperature, and $\eta_{\text {bf }}$ is the beam filling factor, considered to be equal to 1.0 due to the large extension of the core emission compared to the beam size. The second equation relates the optical depth of a given transition to the total column density of the molecule, $N_{\text {col }}$. The line is considered to be Gaussian, with a full width at half maximum equal to $\Delta V$. Here, $h$ is the Planck constant and $k_{\mathrm{B}}$ the Boltzmann constant. Equation (3) depends on several spectroscopic constants: the line frequency $v$, the upper state energy $E_{\mathrm{u}}$ and degenerancy $g_{\mathrm{u}}$, the Einstein coefficient $A_{\mathrm{ul}}$, and the partition function $Q$. This latter equation holds both for an entire rotational transition and for an individual hyperfine component, depending on the splitting considered for the levels in the calculation of the partition function and the corresponding level degeneracy used. We emphasise that Eqs. (2) and (3) represent the integrated form of the (differential) radiative transfer equations. This is the appropriate approach since observations yield essentially integrated quantities (such as column density, fluxes, etc.).

\subsubsection{Column density maps of individual molecules}

To derive the column density of each molecule, we first smooth the angular resolution of the high-frequency transitions to the one with the largest beam. We then fit $N_{\text {col }}$ pixel by pixel, minimising the residuals between the values of $T_{\mathrm{MB}}$ obtained through Eqs. (3) and (2) and the observed peak temperatures 
for all the available transitions. The observed peak temperatures are obtained with a Gaussian fit to the observed spectra, deriving in this way both $T_{\mathrm{MB}}$ and $\Delta V$ at the same time. When the line presents hyperfine structure, we select a single component. The choice of the $T_{\text {ex }}$ value to be used in Eqs. (2) and (3) is made so that this approach at the centre of the core agrees with the $N_{\text {col }}$ value obtained with MOLLIE, within the uncertainties. The $T_{\mathrm{ex}}$ values obtained in this way are used to model the whole map. The chosen method to select $T_{\mathrm{ex}}$ for each line and the assumption that this quantity is spatially constant are discussed in detail in Appendix C. We now describe in detail on which spectral features we focus to fit $N_{\text {col }}$ for each species.

$\mathrm{N}_{2} \mathrm{H}^{+}$. The (1-0) transition of $\mathrm{N}_{2} \mathrm{H}^{+}$presents a single, isolated component at the high-frequency end of its spectrum, which is a perfect candidate for our analysis, also providing reliable values for the line width. On the contrary, the hyperfine structure of the (3-2) line collapses in a very complicated structure, with a strong shoulder on the red side. For this transition we thus use the peak temperature of the observed spectra combined with the $\Delta V$ value obtained from the analysis of the (1-0) transition ${ }^{2}$. For the spectroscopic constants needed in Eq. (3), we selected the brightest hyperfine component according to the spectroscopic catalogues (see Appendix A).

$\mathrm{N}_{2} D^{+}$. The $\mathrm{N}_{2} \mathrm{D}^{+}(1-0)$ transition also presents a single isolated component suitable to our purposes. This is not the case for the other two transitions. For the (2-1) line, we find that the best solution is to consider the brightest feature of the spectra, which is composed by four components separated in total by only $\approx 90 \mathrm{kHz}$ (see Appendix A for details). We fit a single Gaussian to this spectral feature in the observations, and we use the additive property of Eq. (3), according to which $\tau_{v}^{\text {tot }}=\sum_{i} \tau_{v}^{i}$, where $i$ labels the hyperfine components. Finally, in the (3-2) spectra the hyperfine structure is closely blended, and therefore we consider it a single line ${ }^{3}$.

$\mathrm{HC}^{18} \mathrm{O}^{+}$. This is the only molecule for which we have a single transition, and thus we cannot fit $N_{\text {col }}$ using the procedure described above. However, this transition is optically thin, as confirmed by the radiative transfer performed by MOLLIE, which predicts a maximum value of $\tau_{v} \approx 0.20$ at the dust peak. Therefore, we use the optically thin approximation of Eqs. (2) and (3), following Caselli et al. (2002a):

$N_{\text {col }}=\frac{8 \pi W v^{3}}{c^{3} A_{\mathrm{ul}}} \frac{Q\left(T_{\mathrm{ex}}\right)}{J_{v}\left(T_{\mathrm{ex}}\right)-J_{v}\left(T_{\mathrm{bg}}\right)} \frac{\mathrm{e}^{\frac{E_{\mathrm{u}}}{k_{\mathrm{B}} T_{\mathrm{ex}}}}}{g_{\mathrm{u}}\left(\mathrm{e}^{\frac{h \nu}{k_{\mathrm{B}} T_{\mathrm{ex}}}}-1\right)}$,

where $W$ is the integrated intensity of the line. We therefore fit a Gaussian to the spectrum in each pixel; we subsequently compute its integrated intensity and then derive $N_{\text {col }}$ using Eq. (4).

$\mathrm{DCO}^{+}$. The spectra of $\mathrm{DCO}^{+}$are the simplest to analyse since the hyperfine structure due to the deuterium atom is compact if compared to our spectral resolution. We consider the three transitions as single lines, fit a Gaussian profile to each of them, and fit the molecular column density minimising the

\footnotetext{
2 Several other approaches, such as trying to fit a Gaussian avoiding the red-wing component, were tested but proved to be unsuccessful.

3 We tested this hypothesis at the emission peak by comparing our results with those coming from fitting the complete hyperfine structure using GILDAS/CLASS package. We find consistent results within our uncertainties.
}

residuals between the synthetic spectra and the Gaussian fits to the observations.

Figure 8 shows the column density maps obtained for each molecule as described immediately above, while their peak values are summarised in Table 4. Our results are consistent with those of Caselli et al. (2002a), which are quoted to be accurate within a factor of two. We however highlight that a direct comparison with other works is difficult (see Sect. 4). We report in Appendix A the spectroscopic values used for each transition. The values of $T_{\mathrm{ex}}$ that ensure that the above method provides the same peak column density as computed by MOLLIE are reported in the last column of Table 3 . These values confirm that the assumption of a single $T_{\mathrm{ex}}$ value for all transitions is incorrect, since the (3-2) lines present excitation temperatures that are $\approx 2 \mathrm{~K}$ lower than the respective (1-0) ones. For most transitions, $T_{\mathrm{ex}}$ is lower than the gas kinetic temperature, suggesting that many of the detected lines are subthermally excited.

One strong assumption of our method is the line Gaussianity. This neglects any spectral asymmetry due for example to the contraction motions, which are clearly visible in some spectra. We want to emphasise that any improvement of our analysis is strongly related to the development of a better physical model for L1544. In fact, the one-dimensional model of Keto et al. (2015) allows us to solve the radiative transfer problem only at the dust peak, since the fact that L1544 is not spherically symmetric becomes critical moving away from the central point. Since our goal is to investigate the deuteration properties in the whole extended core, we are forced to make further assumptions and simplifications.

Once the column density maps are ready, we can compute the deuterium fraction, dividing the column density of the D-bearing species and that of the main isotopologue pixel by pixel. First, the beam sizes of the maps are matched to allow a fair comparison. The column density of $\mathrm{HCO}^{+}$is derived from that of $\mathrm{HC}^{18} \mathrm{O}^{+}$ using the standard isotopic ratio: $N_{\mathrm{col}}\left(\mathrm{HCO}^{+}\right)=N_{\mathrm{col}}\left(\mathrm{HC}^{18} \mathrm{O}^{+}\right) \times$ 557. Figures 9 and 10 show the resulting images for the $\mathrm{D} / \mathrm{H}$ ratio.

\subsubsection{Evaluation of the uncertainties}

In the approach adopted to compute the molecular column densities, the main source of uncertainty is found in the excitation temperature values. In fact, they depend on several other parameters, such as the assumed physical and chemical models. We choose a conservative approach, and decide that the $T_{\text {ex }}$ values in Table 3 are accurate within $0.5 \mathrm{~K}$. This choice is supported by the following considerations. First of all, such a variation in $T_{\text {ex }}$ translates into an average variation of $20-30 \%$ in the computed column densities, which corresponds to an equal variation of the molecular abundance. We ran MOLLIE again, modifying the abundance profiles by $30 \%$, and the resulting modelled spectra deviate significantly from the observed ones. This means that our data are indeed sensitive to a variation of $0.5 \mathrm{~K}$ in $T_{\mathrm{ex}}$. Furthermore, this variation in $T_{\mathrm{ex}}$ is the limit suggested by our analysis on the possible spatial variation of this quantity (see Appendix C).

The column density maps, computed with the $T_{\mathrm{ex}}$ values modified by $0.5 \mathrm{~K}$, therefore provide the sought-after uncertainties. The uncertainties on the $\mathrm{D} / \mathrm{H}$ ratios are derived through standard error propagation from the ones on the molecular column density. The median relative errors are $41 \%$ for $\mathrm{D} / \mathrm{H}_{\mathrm{N}_{2} \mathrm{H}^{+}}$ and $22 \%$ for $\mathrm{D} / \mathrm{H}_{\mathrm{HCO}^{+}}$. The lower mean uncertainty on the $\mathrm{HCO}^{+}$results depends on how sensitive $N_{\text {col }}$ is to $T_{\text {ex }}$ variations, according to Eq. (3). The column density of $\mathrm{N}_{2} \mathrm{D}^{+}$, for example, 

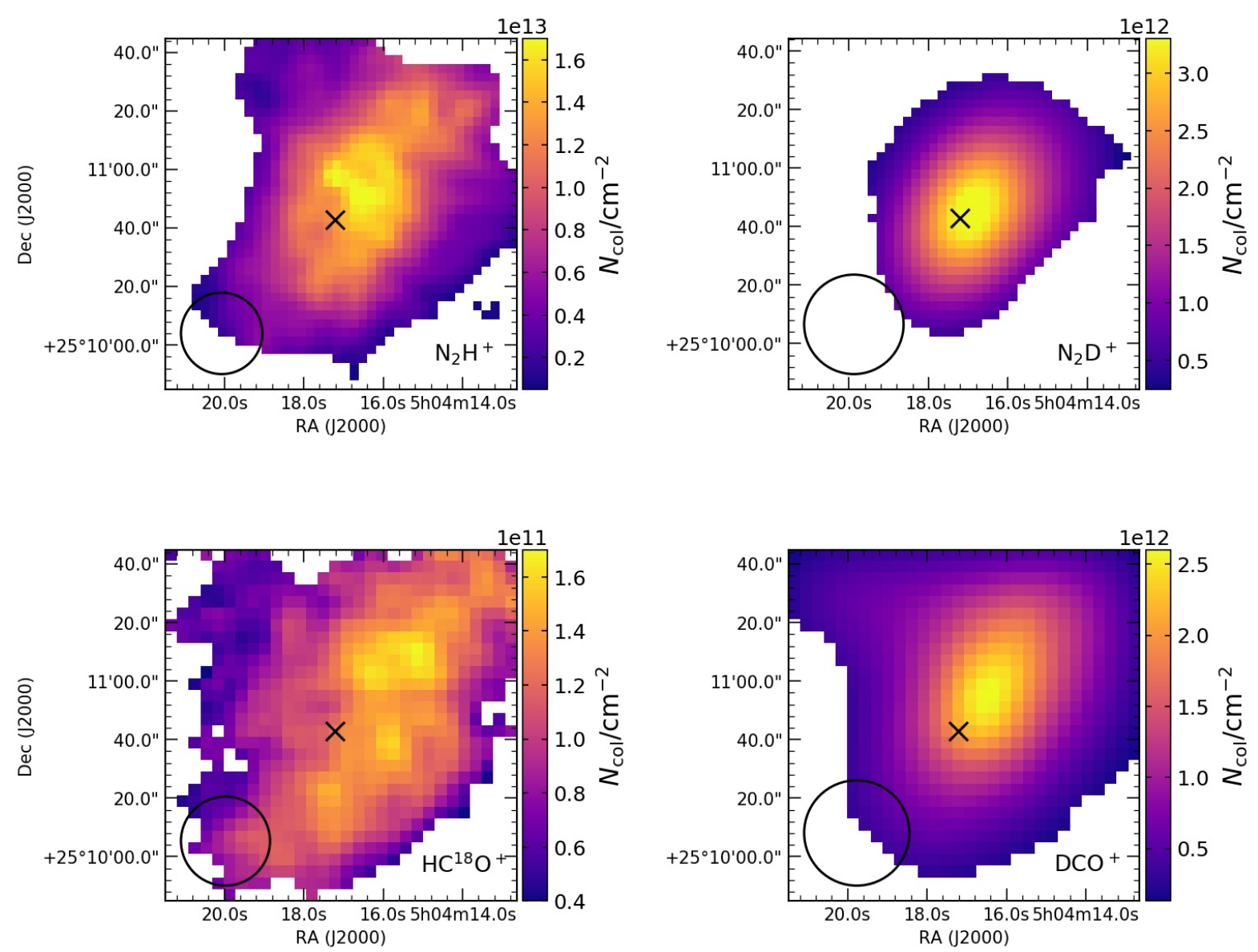

Fig. 8. Column density maps obtained for each molecule (labelled in the bottom-right corner of each panel). The beam sizes are shown in the bottom-left corners. The black cross represents the position of the millimetre dust peak.

Table 4. Peak values of the column densities for the different molecules.

\begin{tabular}{cc}
\hline \hline Molecule & \\
\hline $\mathrm{N}_{2} \mathrm{H}^{+}$ & $1.8_{\text {col }}^{+1.1} \times \mathrm{cm}^{-2}$ \\
$\mathrm{~N}_{2} \mathrm{D}^{+}$ & $3.5_{-1.0}^{+2.0} \times 10^{13}$ \\
$\mathrm{HC}^{18} \mathrm{O}^{+}$ & $1.7_{-0.1}^{+0.1} \times 10^{11}$ \\
$\mathrm{HCO}^{+}$ & $9.5_{-0.6}^{+0.6} \times 10^{13}$ \\
$\mathrm{DCO}^{+}$ & $2.6_{-0.8}^{+1.2} \times 10^{12}$ \\
\hline
\end{tabular}

Notes. ${ }^{(a)}$ The column density of $\mathrm{HCO}^{+}$is derived from the one of $\mathrm{HC}^{18} \mathrm{O}^{+}$using the standard isotopic ratio ${ }^{16} \mathrm{O} /{ }^{18} \mathrm{O}=557$ (see main text).

is more heavily affected than that of $\mathrm{HC}^{18} \mathrm{O}^{+}$by a $T_{\text {ex }}$ change of $0.5 \mathrm{~K}$, and therefore its relative error is higher.

\section{Discussion}

The column density maps presented in Fig. 8 show that different molecules exhibit different morphologies. In particular, one can see differences in the position of the column density maxima with respect to the dust peak. Both $N_{\text {col }}\left(\mathrm{N}_{2} \mathrm{H}^{+}\right)$and $N_{\text {col }}\left(\mathrm{N}_{2} \mathrm{D}^{+}\right)$ peak close to the millimetre dust peak, and while the former has a more extended distribution, the latter is highly concentrated in the densest part of the core (as already noted in Caselli et al. 2002a). The abundance profiles in Fig. 5, despite referring to a one-dimensional core model, confirm these trends. The peak of $\mathrm{N}_{2} \mathrm{H}^{+}$abundance is found at a larger radius with respect to $\mathrm{N}_{2} \mathrm{D}^{+}$.

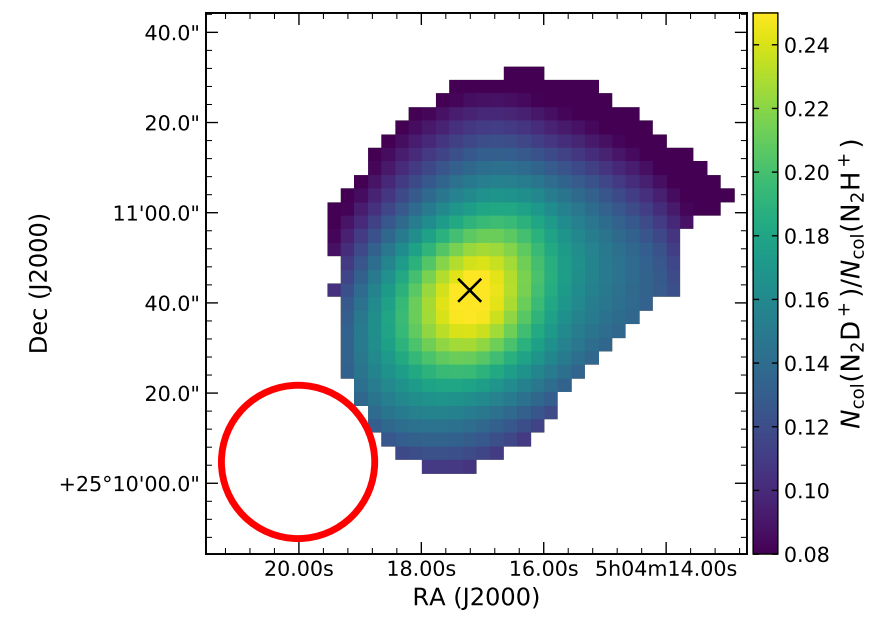

Fig. 9. D/H ratio obtained for $\mathrm{N}_{2} \mathrm{H}^{+}$in L1544. The beam is shown in red, and the black cross represents the dust peak position.

Despite the fact that $\mathrm{N}_{2} \mathrm{D}^{+}$is concentrated in the central region, it is interesting to notice that the chemical model that provides the best fit to its transitions predicts some extent of molecular depletion due to freeze-out, as described in Sect. 3.2.2. This is to our knowledge the first time that $\mathrm{N}_{2} \mathrm{D}^{+}$depletion, which is often predicted by chemical models, is confirmed by observational data. We are aware that our spatial resolution (35" corresponds to $\approx 0.03$ pc at the distance of L1544) is not sufficiently high to investigate this point exhaustively. However, the fact that we need a model with some central depletion to reproduce the observed spectra is clear confirmation. As a further test, 


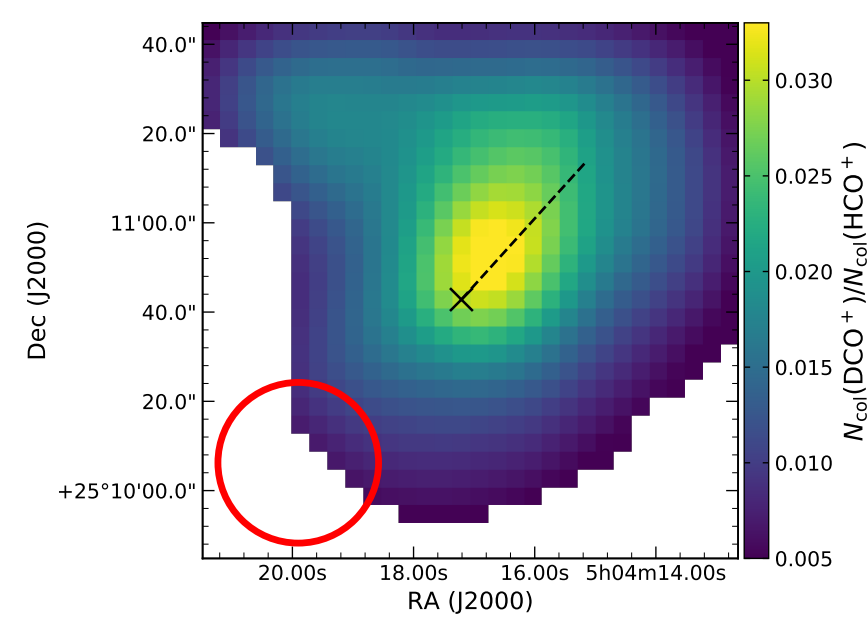

Fig. 10. Deuterium fraction of $\mathrm{HCO}^{+}$obtained in $\mathrm{L} 1544$. The beam is shown in red, and the black cross represents the dust peak position. The dashed line is the cut used to produce Fig. 12.

we tried to model all the $\mathrm{N}_{2} \mathrm{H}^{+}$and $\mathrm{N}_{2} \mathrm{D}^{+}$spectra with MOLLIE using a flat abundance profile with no depletion, which only decreases in the outskirts of the source due to photodissociation. This model is able to accurately reproduce the (1-0) lines, but overestimates the fluxes of the higher-J transitions, which have a higher critical density and are thus sensitive to the abundance in the central part of the core. Furthermore, we derived the molecular column density of $\mathrm{N}_{2} \mathrm{D}^{+}$analysing only the (3-2) transition in the optically thin approximation, using Eq. (4). This allows us to obtain a map of higher resolution, since the beam size at $1 \mathrm{~mm}$ is $\approx 12^{\prime \prime}$ (corresponding to $\approx 0.01 \mathrm{pc}$ at L1544 distance). The results are shown in Fig. 11. Understandably, the absolute values of $N_{\text {col }}$ are different from those obtained via our complete analysis. However, in this case we are more interested in the morphology of the molecular distribution. Figure 11 shows that $\mathrm{N}_{2} \mathrm{D}^{+}$column density is not particularly concentrated around the centre of the core and that it does not peak exactly at this position. Instead, it presents multiple secondary peaks, which are significant with respect to our noise level. These features support our hypothesis of an abundance profile which is not increasing or constant in the innermost part of the core, but that on the contrary is decreasing due to the depletion of the molecule from the gas phase.

Future observational follow-ups with higher resolution will help us to better understand the extent of $\mathrm{N}_{2} \mathrm{H}^{+}$and $\mathrm{N}_{2} \mathrm{D}^{+}$depletion, which is of particular interest since both molecules (and $\mathrm{N}_{2} \mathrm{D}^{+}$in particular) are expected to preferentially trace the central regions of prestellar cores. Moreover, this provides clues on the freeze-out of the parent molecule $\mathrm{N}_{2}$, which is not observable in cold and dense environments. It is also interesting to mention that ammonia, in comparison, does not exhibit any sign of depletion at the centre of L1544 (Crapsi et al. 2007).

The $\mathrm{DCO}^{+}$morphology is also relatively concentrated, similarly to $\mathrm{N}_{2} \mathrm{D}^{+}$, but its peak is shifted in the northwest direction. This is confirmed by the integrated intensity maps shown in the bottom panels of Fig. 3. In particular the integrated intensity map for $\mathrm{DCO}^{+}(3-2)$, which has the best angular resolution $\left(12^{\prime \prime}\right)$, shows that the molecular emission peaks $\approx 15^{\prime \prime}$ away from the dust peak. This morphology is due to the counter-balance of two chemical mechanisms: namely (i) the freeze-out onto dust grains of the C- and O-bearing species, which makes them depleted at very high densities and low temperatures (Caselli et al. 1999; Tafalla et al. 2002); and (ii) the fact that deuterated molecules prefer exactly these physical conditions, as described in Sect. 1.

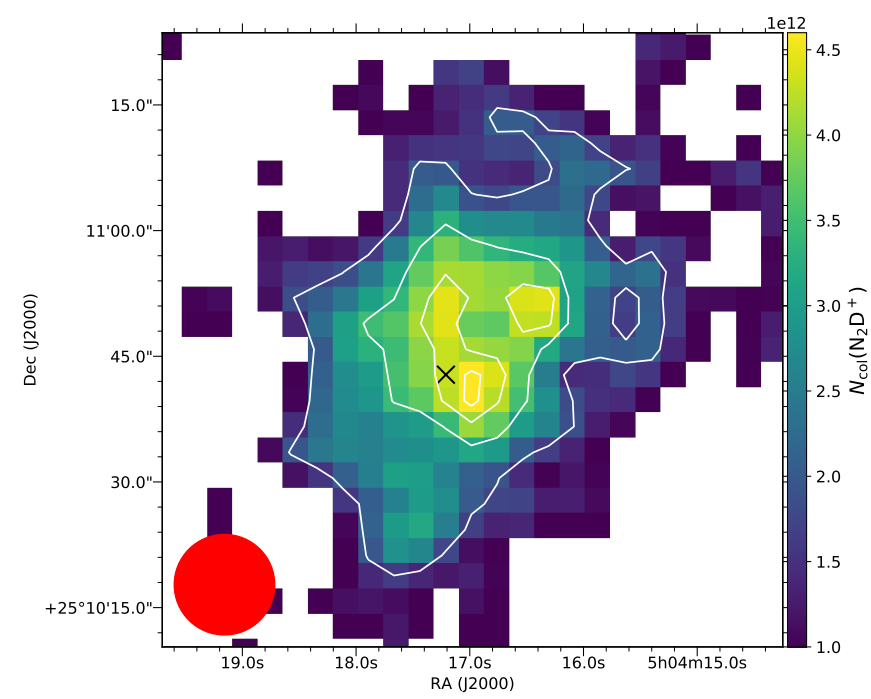

Fig. 11. $\mathrm{N}_{2} \mathrm{D}^{+}$column density obtained analysing only the (3-2) transition, thus obtaining a spatial resolution that is almost three times higher with respect to the corresponding panel in Fig. 8. The white contours represent the integrated intensity of the line, at levels of $[5,9,11,12] \sigma$ $\left(1 \sigma=0.05 \mathrm{~K} \mathrm{~km} \mathrm{~s}^{-1}\right)$.

This explanation additionally gives a straightforward interpretation of the $N\left(\mathrm{HC}^{18} \mathrm{O}^{+}\right)$map. This molecule presents the highest depletion level of those analysed here, as also confirmed by the abundance profile predicted by our chemical network. Its column density peak is found $\approx 35^{\prime \prime}$ away from the dust peak, in the northwest direction.

Another interesting point is that, as described in Sect. 3.2, we could not reproduce the $\mathrm{HCO}^{+}$and $\mathrm{HC}^{18} \mathrm{O}^{+}(1-0)$ spectra using the same abundance profile, simply scaled by the oxygen isotopic ratio. To reproduce the strong blue asymmetry shown by the main isotopologue, we needed to adopt a model with a thick envelope, where the molecule is very abundant $\left(X_{\mathrm{col}}(\mathrm{HCO}+) \gtrsim\right.$ $\left.10^{-7}\right)$. On the contrary, if we use this dense outer layer also for the rare isotopologue, we are not able to correctly fit the spectral profile, since we overestimate the $\mathrm{HC}^{18} \mathrm{O}^{+}$abundance in the envelope. We believe that this is a consequence of the selective photodissociation of $\mathrm{HC}^{18} \mathrm{O}^{+}$. While the main isotopologue is so abundant that it is able to self-shield effectively at large radii, the $\mathrm{HC}^{18} \mathrm{O}^{+}$abundance is not high enough to block the photodissociating photons that can therefore penetrate deeper into the core.

The morphological features previously described for the different column density maps reflect directly on the deuterium fraction maps. Since the D-bearing isotopologues peak closer to the dust peak than the corresponding main species, the $\mathrm{D} / \mathrm{H}$ maps exhibit a compact morphology around the dust peak. The maximum values that we found are $\mathrm{D} / \mathrm{H}_{\mathrm{N}_{2} \mathrm{H}^{+}}=0.26_{-0.14}^{+0.15}$ and $\mathrm{D} / \mathrm{H}_{\mathrm{HCO}^{+}}=0.035_{-0.012}^{+0.015}$, which are in good agreement with previous estimations (see e.g. Caselli et al. 2002a; Crapsi et al. 2005). It is however difficult to make a direct comparison with previous works. For example, Caselli et al. (2002a) used a constant $T_{\text {ex }}$ approach with different excitation temperatures with respect to ours (e.g. $T_{\text {ex }}=5.0 \mathrm{~K}$ for $\mathrm{N}_{2} \mathrm{H}^{+}$and $4.9 \mathrm{~K}$ for $\mathrm{N}_{2} \mathrm{D}^{+}$). These latter study also lacked some important transitions such as $\mathrm{N}_{2} \mathrm{D}^{+}(1-0)$ and $\mathrm{DCO}^{+}(1-0)$, their frequencies being too low for the receivers used at the time.

We find that $\mathrm{N}_{2} \mathrm{H}^{+}$is more deuterated than $\mathrm{HCO}^{+}$, as expected since $\mathrm{N}_{2}$ (and $\mathrm{N}_{2} \mathrm{H}^{+}$in turn) is a late-type molecule and is less affected by depletion (Nguyen et al. 2018). Therefore, $\mathrm{N}_{2} \mathrm{H}^{+}$appears in conditions where $\mathrm{CO}$ is already depleted 


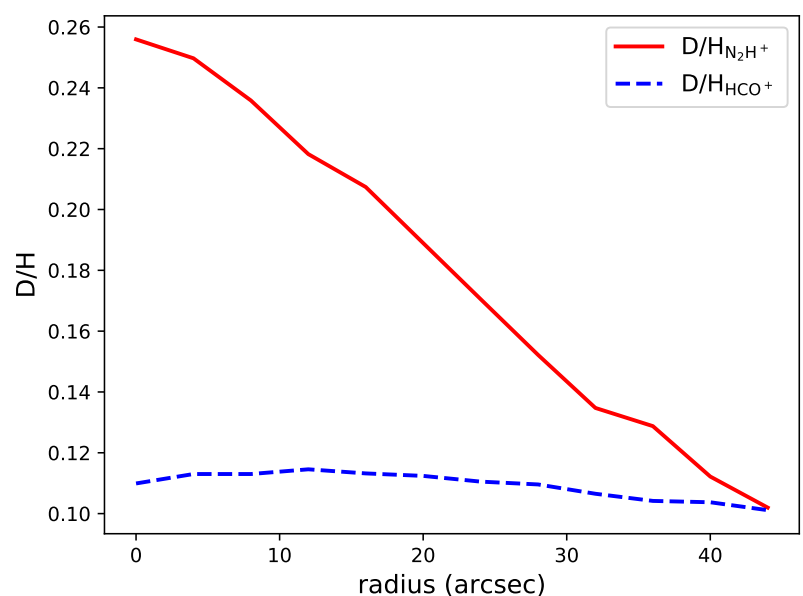

Fig. 12. Comparison of the trends of $\mathrm{D} / \mathrm{H}_{\mathrm{N}_{2} \mathrm{H}^{+}}$(red) and $\mathrm{D} / \mathrm{H}_{\mathrm{HCO}^{+}}$(blue) along the cut shown in Fig. 10. The data points of $\mathrm{HCO}^{+}$have been shifted upwards by 0.10 to allow an easier comparison.

and $\mathrm{H}_{2} \mathrm{D}^{+}$is more abundant. The deuteration level of $\mathrm{N}_{2} \mathrm{H}^{+}$ decreases from 26 to $10 \%$ in $45^{\prime \prime}$, while $\mathrm{D} / \mathrm{H}_{\mathrm{HCO}^{+}}$varies from 3.5 to $2.0 \%$. Figure 12 shows the $\mathrm{D} / \mathrm{H}$ values for the two tracers as a function of radius for a cut crossing the dust peak and the $\mathrm{D} / \mathrm{H}_{\mathrm{HCO}^{+}}$maximum, shown with a dashed line in Fig. 10. The deuteration level thus decreases faster for $\mathrm{N}_{2} \mathrm{H}^{+}$than for $\mathrm{HCO}^{+}$, mainly as a consequence of the fact that $\mathrm{DCO}^{+}$is more extended than $\mathrm{N}_{2} \mathrm{D}^{+}$with respect to their main isotopologues and that $\mathrm{HCO}^{+}$and $\mathrm{DCO}^{+}$are more affected by freeze-out in the core centre compared to $\mathrm{N}_{2} \mathrm{H}^{+}$and $\mathrm{N}_{2} \mathrm{D}^{+}$.

\section{Conclusions}

In this work we performed a detailed analysis of multiple transitions of $\mathrm{N}_{2} \mathrm{H}^{+}, \mathrm{N}_{2} \mathrm{D}^{+}, \mathrm{HC}^{18} \mathrm{O}^{+}, \mathrm{DCO}^{+}$with recent highsensitivity observations, which allow us to investigate the molecular properties with high-S/N data across the whole L1544 core. Using a non-LTE approach combined with the molecular abundances computed with chemical models, we derived the excitation conditions of the molecules at the dust peak. Our resulting $T_{\text {ex }}$ values confirm that the assumption of a common excitation temperature for different rotational transitions or even for different hyperfine components does not hold for these molecular ions. The molecular abundances derived with our chemical models are in general not able to reproduce the observations, especially for $\mathrm{N}_{2} \mathrm{H}^{+}$and $\mathrm{N}_{2} \mathrm{D}^{+}$for which we had to increase the predicted abundance by up to $300 \%$ to obtain a good fit to the observed spectra. Despite these discrepancies, we are able to draw some important conclusions.

All the analysed molecules show some level of depletion in the very central parts of the core due to freeze-out onto the dust grains. This phenomenon is most prominent for $\mathrm{HCO}^{+}$and its isotopologues, but it is seen also for $\mathrm{N}_{2} \mathrm{H}^{+}$and to a lesser extent for $\mathrm{N}_{2} \mathrm{D}^{+}$. This is of particular interest because it is the first time that $\mathrm{N}_{2} \mathrm{D}^{+}$depletion, which is often predicted by chemical models, finds confirmation in observational data. Further higherresolution observations will help us investigate this point. The $\mathrm{D} / \mathrm{H}$ ratios are peaked very close to the dust peak of $\mathrm{L} 1544$, and show different values, the deuteration of $\mathrm{N}_{2} \mathrm{H}^{+}$being higher than that of $\mathrm{HCO}^{+}$(26 and $3.5 \%$, respectively). In an upcoming paper, we will use the results derived here to obtain spatially resolved information on the ionisation degree, a key parameter in the early stages of star formation.
Acknowledgements. The authors thank the anonymous referee, whose comments helped to increase the quality of the manuscript. E.R. thanks Dr. Jacob Laas for his help in checking the overall language quality.

\section{References}

Amano, T., Hirao, T., \& Takano, J. 2005, J. Mol. Spectr., 234, 170

André, P., Di Francesco, J., Ward-Thompson, D., et al. 2014, in Protostars and Planets VI, eds. H. Beuther, R. S. Klessen, C. P. Dullemond, \& T. Henning (Tucson, AZ: University of Arizona Press), 27

Bacmann, A., Lefloch, B., Ceccarelli, C., et al. 2002, A\&A, 389, L6

Bizzocchi, L., Caselli, P., Leonardo, E., \& Dore, L. 2013, A\&A, 555, A109

Caselli, P. 2002, Planet. Space Sci., 50, 1133

Caselli, P., \& Dore, L. 2005, A\&A, 433, 1145

Caselli, P., Walmsley, C. M., Terzieva, R., \& Herbst, E. 1998, ApJ, 499, 234

Caselli, P., Walmsley, C. M., Tafalla, M., Dore, L., \& Myers, P. C. 1999, ApJ, 523, L165

Caselli, P., Walmsley, C. M., Zucconi, A., et al. 2002a, ApJ, 565, 344

Caselli, P., Walmsley, C. M., Zucconi, A., et al. 2002b, ApJ, 565, 331

Caselli, P., Keto, E., Bergin, E. A., et al. 2012, ApJ, 759, L37

Caselli, P., Bizzocchi, L., Keto, E., et al. 2017, A\&A, 603, L1

Cazzoli, G., Cludi, L., Buffa, G., \& Puzzarini, C. 2012, ApJS, 203, 11

Ceccarelli, C., Caselli, P., Bockelée-Morvan, D., et al. 2014, in Protostars and Planets VI, eds. H. Beuther, R. S. Klessen, C. P. Dullemond, \& T. Henning (Tucson, AZ: University of Arizona Press), 859

Chacon-Tanarro, A., Pineda, J. E., Caselli, P., et al. 2019, A\&A, 623, A118

Crapsi, A., Caselli, P., Walmsley, C. M., et al. 2005, ApJ, 619, 379

Crapsi, A., Caselli, P., Walmsley, M. C., \& Tafalla, M. 2007, A\&A, 470, 221

Dalgarno, A. 2006, Proc. Natl. Acad. Sci., 103, 12269

Dalgarno, A., \& Lepp, S. 1984, ApJ, 287, L47

Daniel, F., Cernicharo, J., \& Dubernet, M.-L. 2006, ApJ, 648, 461

Daniel, F., Cernicharo, J., Roueff, E., Gerin, M., \& Dubernet, M. L. 2007, ApJ, 667, 980

Daniel, F., Gérin, M., Roueff, E., et al. 2013, A\&A, 560, A3

Dore, L., Caselli, P., Beninati, S., et al. 2004, A\&A, 413, 1177

Doty, S. D., Schöier, F. L., \& van Dishoeck, E. F. 2004, A\&A, 418, 1021

Flower, D. R. 1999, MNRAS, 305, 651

Goldsmith, P. F., \& Langer, W. D. 1999, ApJ, 517, 209

Guelin, M., Langer, W. D., Snell, R. L., \& Wootten, H. A. 1977, ApJ, 217, L165

Hily-Blant, P., Walmsley, M., Pineau Des Forêts, G., \& Flower, D. 2010, A\&A, 513, A41

Keto, E. R. 1990, ApJ, 355, 190

Keto, E., \& Caselli, P. 2008, ApJ, 683, 238

Keto, E., \& Caselli, P. 2010, MNRAS, 402, 1625

Keto, E., Rybicki, G. B., Bergin, E. A., \& Plume, R. 2004, ApJ, 613, 355

Keto, E., Caselli, P., \& Rawlings, J. 2015, MNRAS, 446, 3731

Kong, S., Caselli, P., Tan, J. C., Wakelam, V., \& Sipilä, O. 2015, ApJ, 804, 98

Lattanzi, V., Walters, A., Drouin, B. J., \& Pearson, J. C. 2007, ApJ, 662, 771

Lee, C. W., Myers, P. C., \& Tafalla, M. 2001, ApJS, 136, 703

Linsky, J. L. 2003, Space Sci. Rev., 106, 49

Lique, F., Daniel, F., Pagani, L., \& Feautrier, N. 2015, MNRAS, 446, 1245

McKee, C. F. 1989, ApJ, 345, 782

Mouschovias, T. C., \& Spitzer, L. J. 1976, ApJ, 210, 326

Nguyen, T., Baouche, S., Congiu, E., et al. 2018, A\&A, 619, A111

Pagani, L., Salez, M., \& Wannier, P. G. 1992, A\&A, 258, 479

Pagani, L., Bacmann, A., Cabrit, S., \& Vastel, C. 2007, A\&A, 467, 179

Pagani, L., Bourgoin, A., \& Lique, F. 2012, A\&A, 548, L4

Punanova, A., Caselli, P., Pineda, J. E., et al. 2018, A\&A, 617, A27

Redaelli, E., Bizzocchi, L., Caselli, P., et al. 2018, A\&A, 617, A7

Schlafly, E. F., Green, G., Finkbeiner, D. P., et al. 2014, ApJ, 786, 29

Shirley, Y. L. 2015, PASP, 127, 299

Sipilä, O., Caselli, P., \& Harju, J. 2015a, A\&A, 578, A55

Sipilä, O., Harju, J., Caselli, P., \& Schlemmer, S. 2015b, A\&A, 581, A122

Sipilä, O., Caselli, P., Redaelli, E., Juvela, M., \& Bizzocchi, L. 2019, MNRAS, 487, 1269

Spezzano, S., Bizzocchi, L., Caselli, P., Harju, J., \& Brünken, S. 2016, A\&A, 592, L11

Spezzano, S., Caselli, P., Bizzocchi, L., Giuliano, B. M., \& Lattanzi, V. 2017, A\&A, 606, A82

Tafalla, M., Mardones, D., Myers, P. C., et al. 1998, ApJ, 504, 900

Tafalla, M., Myers, P. C., Caselli, P., Walmsley, C. M., \& Comito, C. 2002, ApJ, 569,815

Ward-Thompson, D., Motte, F., \& Andre, P. 1999, MNRAS, 305, 143

Wilson, T. L. 1999, Rep. Prog. Phys., 62, 143

Yu, S., Pearson, J. C., Drouin, B. J., et al. 2015, J. Mol. Spectr., 314, 19 


\section{Appendix A: The spectroscopic constants}

Table A.1. Spectroscopic values for the transitions used in the analysis to derive the molecular column densities.

\begin{tabular}{|c|c|c|c|c|c|c|}
\hline Rot. transition & Hyperfine comp. ${ }^{(a)}$ & $v(\mathrm{GHz})$ & $g_{\mathrm{u}}$ & $E_{\mathrm{u}} / k_{\mathrm{B}}(\mathrm{K})$ & $A_{\mathrm{ul}} / 10^{-5}\left(\mathrm{~s}^{-1}\right)$ & Reference \\
\hline \multicolumn{7}{|c|}{$\mathrm{N}_{2} \mathrm{H}^{+}$} \\
\hline$J=1-0$ & $J, F_{1}, F=1,0,1 \leftarrow 0,1, *^{(b)}$ & 93.1762604 & 3 & 4.472 & 3.628 & 1 \\
\hline$J=3-2$ & $J, F_{1}, F=3,4,5 \leftarrow 2,3,4$ & 279.5118572 & 11 & 13.41 & $1.25910^{2}$ & 1 \\
\hline \multicolumn{7}{|c|}{$\mathrm{N}_{2} \mathrm{D}^{+}$} \\
\hline$J=1-0$ & $J, F_{1}, F=1,0,1 \leftarrow 0,1, *$ & 77.1121207 & 3 & 3.701 & 2.057 & 2 \\
\hline$J=2-1$ & $J, F_{1}, F=2,3,3 \leftarrow 1,2,2$ & 154.2170958 & 7 & 11.10 & 18.08 & 2 \\
\hline$J=2-1$ & $J, F_{1}, F=2,3,2 \leftarrow 1,2,1$ & 154.2171220 & 5 & 11.10 & 15.63 & 2 \\
\hline$J=2-1$ & $J, F_{1}, F=2,2,3 \leftarrow 1,1,2$ & 154.2171273 & 7 & 11.10 & 14.17 & 2 \\
\hline$J=2-1$ & $J, F_{1}, F=2,3,4 \leftarrow 1,2,3$ & 154.2171888 & 9 & 11.10 & 19.74 & 2 \\
\hline$J=3-2$ & - & 231.3218611 & 7 & 22.20 & 71.38 & 2 \\
\hline \multicolumn{7}{|c|}{$\mathrm{DCO}^{+}$} \\
\hline$J=1-0$ & - & 72.0393123 & 3 & 3.457 & 2.206 & 3 \\
\hline$J=2-1$ & - & 144.0772854 & 5 & 10.37 & 21.18 & 3 \\
\hline$J=3-2$ & - & 216.1125804 & 7 & 20.74 & 76.58 & 3 \\
\hline \multicolumn{7}{|c|}{$\mathrm{HC}^{18} \mathrm{O}^{+}$} \\
\hline$J=1-0$ & - & 85.1622231 & 3 & 4.087 & 3.645 & 4 \\
\hline
\end{tabular}

Notes. ${ }^{(a)}$ When no hyperfine component is indicated, the transition is intended as a single transition. ${ }^{(b)}$ The notation $J, F_{1}, F \leftarrow J^{\prime}, F_{1}^{\prime}, *$ indicates that there are multiple but degenerate lower states, which have the same energy but different $F$ quantum number.

References. The spectroscopic data are taken from: (1) our calculation based on data from Cazzoli et al. (2012), (2) our calculation based on data from Dore et al. (2004), Amano et al. (2005), and Yu et al. (2015), (3) our calculation based on data from Caselli \& Dore (2005) and Lattanzi et al. (2007), and (4) from Bizzocchi et al. (in prep.).

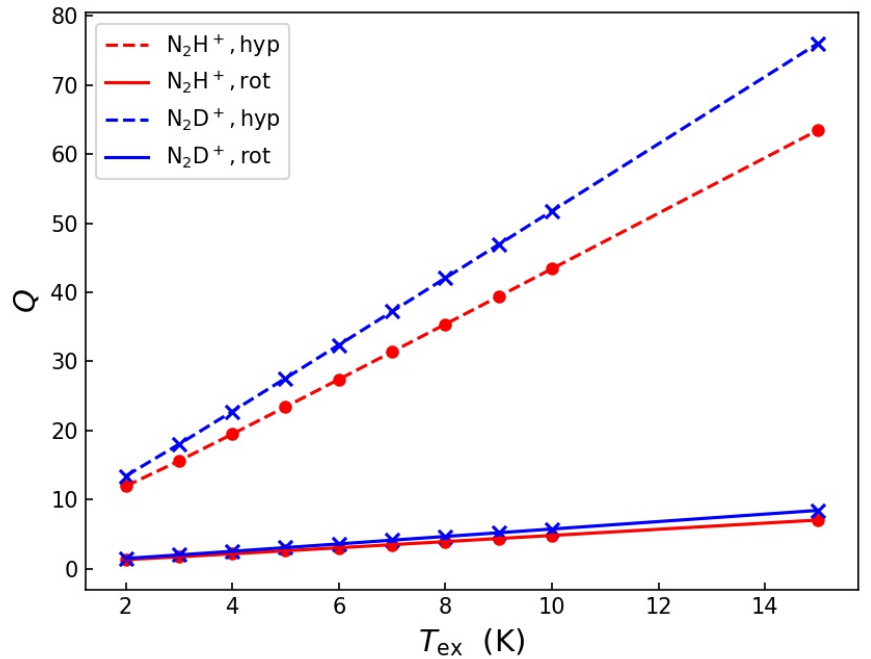

Fig. A.1. Partition function of $\mathrm{N}_{2} \mathrm{H}^{+}$(red circles) and $\mathrm{N}_{2} \mathrm{D}^{+}$(blue crosses) as a function of excitation temperature. $Q$ for rotational transition, neglecting the hyperfine structure, are indicated with solid lines, while the dashed curves take into account all the hyperfine levels.

In Table A.1 we report the values of the spectroscopic parameters used to derive the column densities, according to the method explained in Sect. 3.3. The references for the shown values are reported in the last column of the table. Figures A.1 and A.2 show the partition function for $\mathrm{N}_{2} \mathrm{H}^{+}$and $\mathrm{HCO}^{+}$(and isotopologues), respectively. In the considered range of temperatures, the correlation is almost linear.

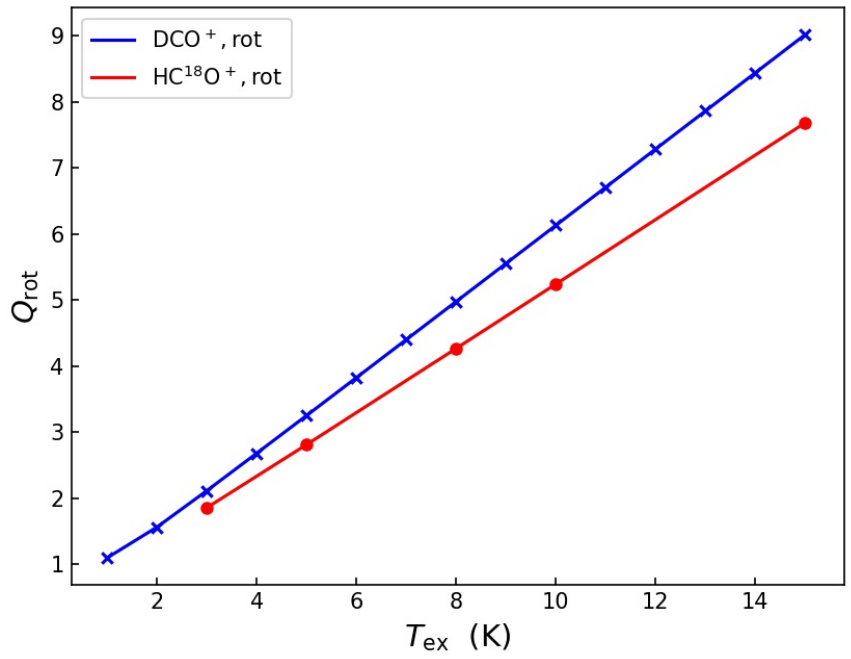

Fig. A.2. Rotational partition function of $\mathrm{DCO}^{+}$(blue crosses) and $\mathrm{HC}^{18} \mathrm{O}^{+}$(red dots), as a function of $T_{\mathrm{ex}}$.

\section{Appendix B: Molecular abundance profiles}

Here, we plot the abundance profiles computed with our chemical network and tested with the non-LTE approach. Figures B.1-B.3 show the models at the five different time-steps, respectively, for $A_{\mathrm{V}}=1,2$, and 5 . 

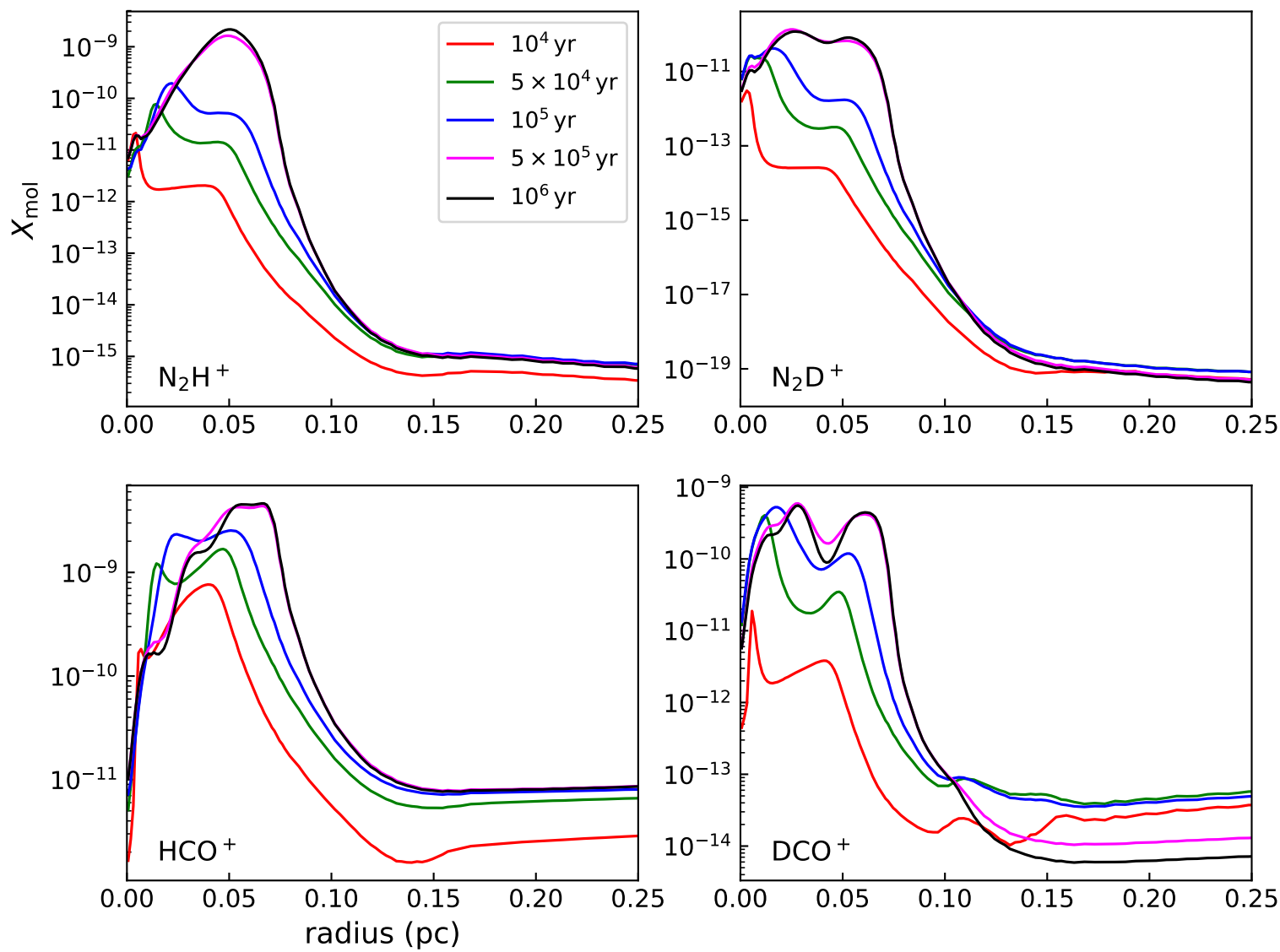

Fig. B.1. Molecular abundances in the model with $A_{\mathrm{V}}=1$ mag for $\mathrm{N}_{2} \mathrm{H}^{+}$(top left), $\mathrm{N}_{2} \mathrm{D}^{+}$(top right), $\mathrm{HCO}^{+}$(bottom left), and $\mathrm{DCO}^{+}$(bottom right). The colours represent the different time-steps: $10^{4} \mathrm{yr}$ (red), $5 \times 10^{4} \mathrm{yr}$ (green), $10^{5} \mathrm{yr}$ (blue), $5 \times 10^{5} \mathrm{yr}$ (purple), and $10^{6} \mathrm{yr}(\mathrm{black}$ ).
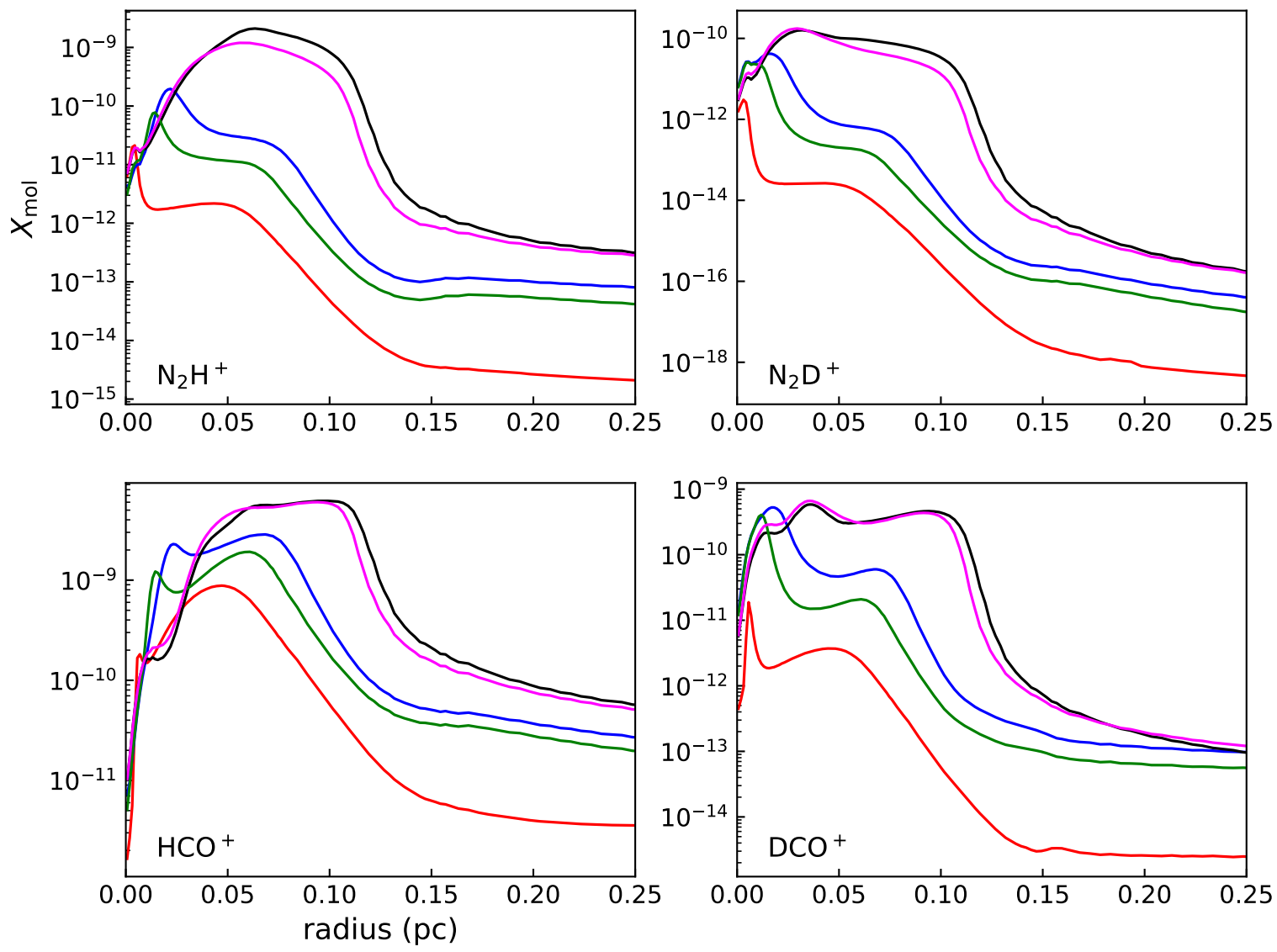

Fig. B.2. Same as Fig. B.1, but for $A_{\mathrm{V}}=2 \mathrm{mag}$.

A15, page 14 of 16 

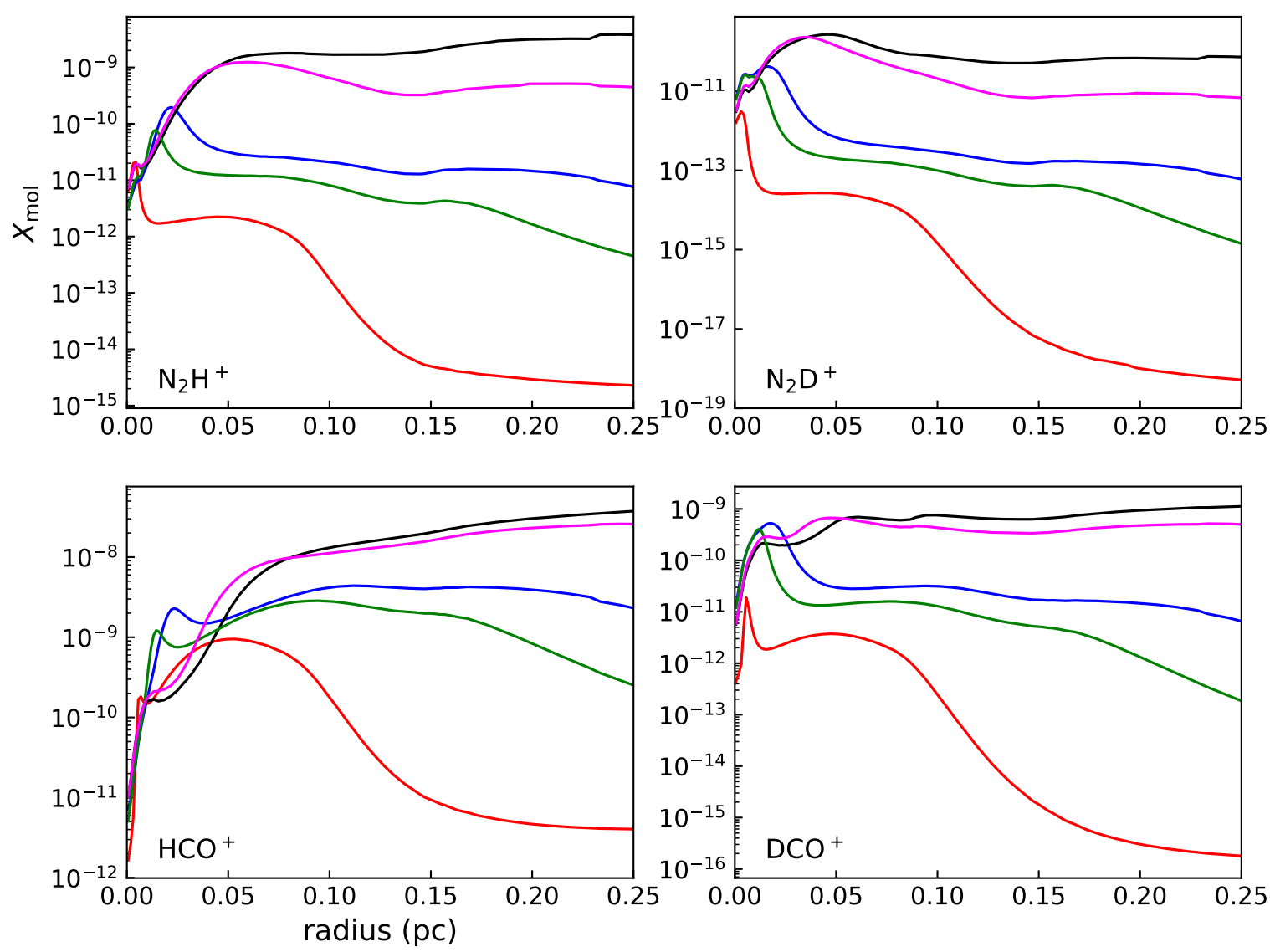

Fig. B.3. Same as Fig. B.1, but for $A_{\mathrm{V}}=5 \mathrm{mag}$.

\section{Appendix C: Discussion on the excitation temperature}

As described in Sect. 3, a main issue to derive the molecular column density when its rotational lines are observed consists in determining the excitation temperatures of the transitions. When $T_{\text {ex }}$ is not the same for all the lines (or when just one line is available), the problem becomes degenerate. A non-LTE analysis is therefore necessary to constrain these parameters $\left(N_{\mathrm{col}}\right.$ and $\left.T_{\mathrm{ex}}\right)$ independently.

One could argue that MOLLIE can be used to derive the $T_{\text {ex }}$ values directly. This is only partially true. MOLLIE, as in any non-LTE radiative transfer code that performs ray tracing, does not operate directly with the excitation temperature quantity; it propagates and counts photons, and it solves the statistical equilibrium equations for the energy level populations. The latter are indeed related to $T_{\text {ex }}$ through the Boltzmann equation. However, the $T_{\mathrm{ex}}$ obtained in this way are only local values, since they can be computed cell by cell ${ }^{4}$. The result is therefore a one-dimensional radial profile of $T_{\mathrm{ex}}$ for each transition.

On the other hand, the $T_{\mathrm{ex}}$ that appears in the equations of radiative transfer is an average quantity, integrated along the line of sight and over the beam size, which relates the observed fluxes with the molecular column density for the beam of that specific observation. It is not straightforward to link the local $T_{\text {ex }}$ profiles obtained in MOLLIE with the $T_{\text {ex }}$ values needed to compute $N_{\text {col }}$, especially because $T_{\text {ex }}$ is an intensive quantity. An approach

\footnotetext{
4 In our simulation, the finest spatial grid has a resolution of $3 \times$ $10^{-3} \mathrm{pc}$, orders of magnitude smaller than the resolution of the observed spectra.
}

that we have tested is to integrate the $T_{\text {ex }}$ profiles along the line of sight, using the $\mathrm{H}_{2}$ column density as a weighting function. Since $\mathrm{H}_{2}$ is the main collisional partner, this choice seems reasonable. However, it neglects other important parameters that affect $T_{\mathrm{ex}}$, such as the gas temperature and the density of the molecule itself. Another point that must be taken into account is that, contrary to $T_{\text {ex }}$, the molecular abundance is an extensive quantity that can be multiplied by $n\left(\mathrm{H}_{2}\right)$ and integrated along the line-of-sight to derive the molecular column density without any ambiguity. It is a natural requirement that the two methods to derive $N_{\mathrm{col}}$, i.e. the physical and chemical modelling done with MOLLIE and the solution of Eqs. (2) and (3), must be consistent and provide the same result at the centre of the core. With this constraint, $T_{\text {ex }}$ becomes a free parameter that can be tuned to ensure the required consistency. This is the selected method to derive the $T_{\text {ex }}$ values reported in Table 3 .

We reiterate that this approach is possible only at the dust peak, because the physical and chemical models are spherically symmetric and cannot reproduce the asymmetries of the core that the observations unveil. Furthermore, the model of Keto et al. (2015) has been specifically developed to reproduce the dust peak.

A strong assumption that we make in Sect. 3.3 is that the $T_{\text {ex }}$ values found, as described immediately above, are constant throughout our map coverage. This is questionable, since one might expect molecules to be less excited in the outskirts of the core, where the gas is less dense. In order to investigate this point, we tried to derive the $\mathrm{DCO}^{+}$column density using a decreasing $T_{\text {ex }}$ profile for each line. $\mathrm{DCO}^{+}$is a representative case, because it has a more extended distribution than for instance $\mathrm{N}_{2} \mathrm{D}^{+}$and three transitions are available at the same 

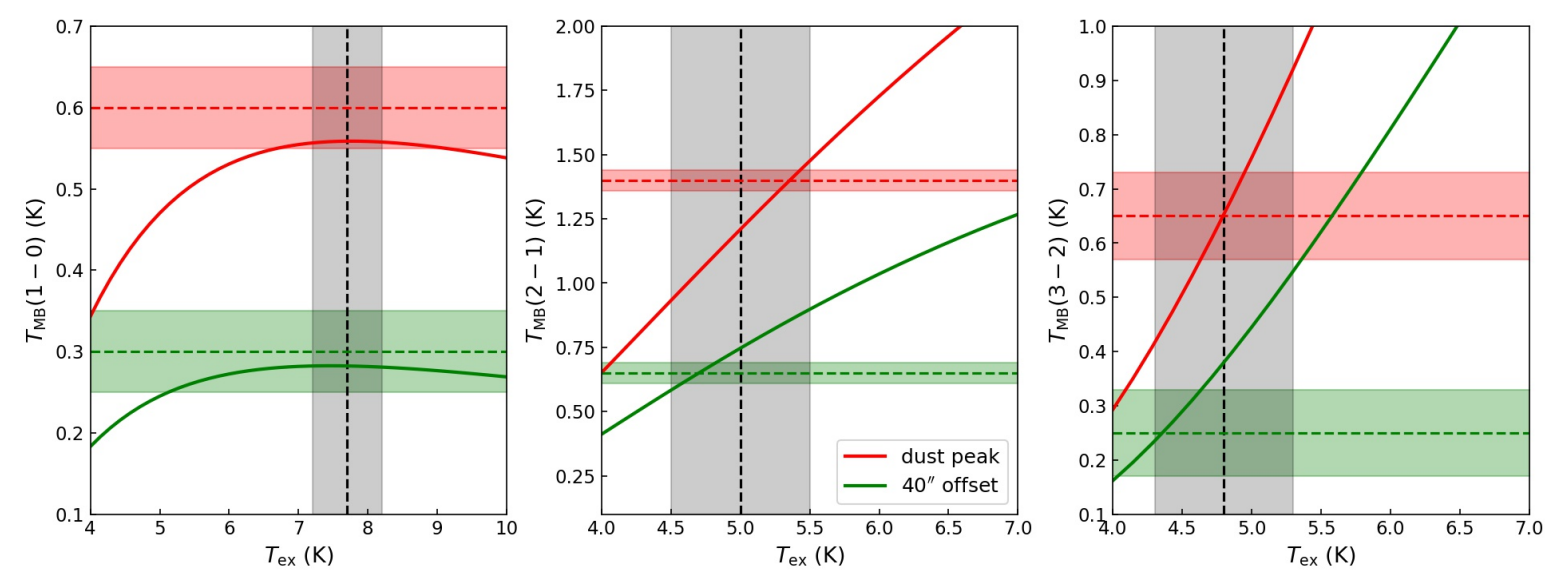

Fig. C.1. Isolated component of $\mathrm{N}_{2} \mathrm{D}^{+}(1-0)$ (left panel), the central component of $\mathrm{N}_{2} \mathrm{D}^{+}(2-1)$ (centre), and the $\mathrm{N}_{2} \mathrm{D}^{+}(3-2)$ line (right panel). In each panel, the horizontal dashed lines are the observed peak temperatures of the selected $\mathrm{N}_{2} \mathrm{D}^{+}$transition at the dust peak (red), and at $40^{\prime \prime}$ of offset (green). The shadowed areas represent observational uncertainties. The solid curves indicate $T_{\mathrm{MB}}$ as a function of $T_{\mathrm{ex}}$, obtained via the radiative transfer equations and using the column density values predicted by MOLLIE at the two offsets. The vertical dashed line is the $T_{\text {ex }}$ value used in the analysis of the maps, and the grey shaded area is its uncertainty $(0.5 \mathrm{~K})$.

time. We realised that a decrease of more than $0.5-1.0 \mathrm{~K}$ within $\approx 60^{\prime \prime}$ (the limit of our map coverage) is enough to cause our $N_{\text {col }}$ fitting routine to break down. With these profiles, the resulting $N_{\text {col }}$ maps present unphysical features, such as values at the border of the core that are several times higher than at the integrated intensity peak, and/or unrealistic morphologies.

In order to further test this assumption, we focus on $\mathrm{N}_{2} \mathrm{D}^{+}$. As already mentioned, MOLLIE and our physical and chemical models are not able to reproduce the observed spectra outside the dust peak. However, $\mathrm{N}_{2} \mathrm{D}^{+}$is sufficiently centrally concentrated and symmetric around the centre of the core to allow a reasonable fit to the observations also outside dust peak. In particular, we were able to compare the synthetic spectra extracted from the model at $40^{\prime \prime}$ offset with the observed signals obtained by averaging the data in a ring at a distance of $40^{\prime \prime}$ from the centre of the core. We can therefore constrain $N_{\mathrm{col}}$ at two different offsets, and verify that the $T_{\mathrm{ex}}$ values that reproduce these column densities are equal within the uncertainties. Figure C.1 shows the results of this analysis. The red and green dashed lines represent the $T_{\mathrm{MB}}$ observed values of the line components used to derive $N_{\text {col }}$ (see
Sect. 3.3.1), at the dust peak and at $40^{\prime \prime}$ offset, respectively. The shaded area indicates the spectra $\mathrm{rms}^{5}$. The solid curves indicate the $T_{\mathrm{MB}}$ of each line as a function of $T_{\mathrm{ex}}$, obtained using Eqs. (2) and (3) using the column density values predicted by MOLLIE at the two offsets $\left(3.5\right.$ and $1.6 \times 10^{12} \mathrm{~cm}^{-2}$, respectively). Finally, the black dashed lines show the $T_{\text {ex }}$ values adopted in this work, and the grey shadows indicate a $0.5 \mathrm{~K}$ variation of this quantity. All the observations are smoothed at the same beam size ( $\left.34^{\prime \prime}\right)$, which is the resolution of the final $N_{\text {col }}$ map. For all three transitions the solid lines cross the interceptions of the shaded areas, suggesting that within our uncertainties we are not able to detect a significant change of $T_{\text {ex }}$.

We want to highlight that our analysis does not imply that $T_{\text {ex }}$ is indeed constant across the whole core, but only that our observations are not sensitive to its variation and that they can be analysed assuming a constant value. Most likely, this is also due to the limited coverage of our maps, which are $2^{\prime} \times 2^{\prime}$ in size, meaning that in a core radius we have at most two or three independent beams. All this said, we assume that our $T_{\text {ex }}$ values reported in Table 3 are accurate to within $0.5 \mathrm{~K}$.
These uncertainties are lower limits, since they do not take into account the calibration uncertainties (up to $15 \%$ ). 